\title{
Ethnobotanique des plantes médicinales anti hémorroïdaires des marchés et villages du Centre et du Littoral Cameroun
}

\author{
Siegfried Didier DIBONG *(1,2), Patrice Brice MVOGO OTTOU1, Deli VANDI², Rosette Christelle \\ NDJIB ${ }^{1}$, Fabrice MONKAM TCHAMAHA ${ }^{1}$, Emmanuel MPONDO MPONDO $(2,3)$ \\ 1 Département de Biologie des Organismes Végétaux, Faculté des Sciences, B.P. 24157 Douala, \\ Cameroun \\ 2 Département des Sciences Pharmaceutiques, Faculté de Médecine et des Sciences Pharmaceutiques, \\ Université de Douala, B.P. 2701 Douala, Cameroun \\ 3 Département de Pharmacotoxicologie et Pharmacocinétique, Faculté de Médecine et des Sciences \\ Biomédicales, Université de Yaoundé I, BP 1364 Yaoundé, Cameroun \\ Auteur de la correspondance : patricemvogoottou@ymail.com
}

Original submitted in on 22nd October 2015. Published online at www.m.elewa.org on 31st December 2015 http://dx.doi.org/10.4314/jab.v96i1.5

\section{RESUME}

Objectif : Très peu de données sont disponibles sur les plantes anti hémorroïdaires en Afrique. Le but est de valoriser la médecine à base des plantes dans le traitement des pathologies hémorroïdaires.

Méthodologie et résultats: Des enquêtes ethnobotaniques ont été réalisées auprès des guérisseurs des villages et des marchés des Régions du Centre et du Littoral. Un dendrogramme évaluant la similitude des connaissances des informateurs a été produit et les Fréquences Relatives (FRs) ainsi que les Facteurs de Consensus d'Utilisation des espèces (ICFs) ont été calculés. Au total, 60 espèces médicinales ont été recensées parmi lesquelles 45 ligneux et 15 herbacées. Elles sont reparties dans 41 familles dont la plus représentée est celle des Fabaceae. Ces espèces, endémiques pour la plupart sont riches en composés phénoliques et sont utilisées en association pour la préparation de 42 recettes dans lesquelles les écorces sont les organes végétaux les plus sollicités. La décoction à l'eau et la boisson prise deux fois par jour plus le bain de siège pris une fois par jour sont les modes de préparation et d'administration des dites recettes. Aucune espèce n'a atteint la valeur moyenne de consensus $(0,5)$ ainsi que la $F R$ moyenne $(50 \%)$. Piper umbellatum, Baillonella toxisperma, Antrocaryon klaineanum, Irvingia gabonensis, Cylicodiscus gabonensis, Mangifera indica, Pycnanthus angolensis, Morinda lucida, Capsicum frutescens, Myrianthus arboreus et Elaeis guineensis sont les espèces ayant atteint un ICF $\geq 0,10$ et une $F R \geq 10 \%$.

Conclusion et application des résultats : La flore camerounaise est riche en espèces de plantes médicinales anti hémorroïdaires et le coût réduit des recettes serait pour les populations démunies, une alternative dans la lutte contre la pauvreté.

Mots clés : Composés phénoliques, Hémorroïdes, ICF et FR, Plantes médicinales, Recettes. 


\section{ABSTRACT}

Objective: There is very little data available on anti-hemorrhoidal plants in Africa. The aim is to promote the use of herbal medicine plants in the treatment of hemorrhoidal diseases.

Methodology and results: Ethnobotanical surveys were conducted with traditional healers in villages and markets of the Centre and Littoral regions. A dendrogram was produced using the Relative Frequencies (RFs) and the Informants Consensus Factors (ICFs) of species. A total of 60 medicinal plant species were recorded including 45 woody and 15 herbaceous. They are distributed in 41 families and the most represented is that of Fabaceae. These species, most of which are endemic, are rich in phenolic compounds and are used in association for the preparation of 42 recipes in which barks are the most requested plant organs. A water decoction and a drink taken twice a day added to a sit bath once a day were the preparation and administration modes of these receipts respectively. No species has reached the average value of consensus $(0.5)$ and the average RF (50\%). Piper umbellatum, Baillonella toxisperma, Antrocaryon klaineanum, Irvingia gabonensis, Cylicodiscus gabonensis, Mangifera indica, Pycnanthus angolensis, Morinda lucida, Capsicum frutescens, Myrianthus arboreus and Elaeis guineensis are the species that have achieved an ICF $\geq 0.10$ and a $R F \geq 10 \%$.

Conclusion and application of results: The Cameroonian flora is rich in anti hemorrhoidal medicinal plant species and their reduced cost could be an alternative in the fight against poverty.

Keywords: Hemorrhoids, ICFs and FRs, Medicinal plants, Phenolic compounds

\section{INTRODUCTION}

Les hémorroïdes sont des formations vasculaires normales présentes chez le fœtus dès la 28ème semaine et dont la fonction est de contribuer à la continence anale (Morgado et al., 1988). La pathogénie de la maladie hémorroïdaire n'est pas clairement définie, mais semble multifactorielle. Les facteurs mécaniques tels que le relâchement de l'appareil musculoligamentaire de suspension, l'altération des moyens de fixation des plexus hémorroïdaires et les facteurs vasculaires tels que l'augmentation de la pression au niveau des coussinets de Thomson, les modifications anatomiques vasculaires sont décrits comme des mécanismes par lesquels les causes de la maladie déclenchent l'évolution de celle-ci (Haas et al., 1984 ; Miller et al., 1988). Les examens cliniques permettent de classer les hémorroïdes en 4 stades évolutifs. Le STADE I correspond à une simple dilatation veineuse à l'effort (hémorroïdes non procidences se manifestant par des rectorragies et une poussée congestive). Le STADE II se caractérise par la tendance à la procidence à l'effort avec réduction spontanée), le STADE III caractérisé par la procidence vraie intermittente avec ou sans réduction digitale et le STADE IV par une procidence permanente et irréductible (Senéjoux, 2010). Maladies de la civilisation, l'affection hémorroïdaire constitue la pathologie la plus fréquente de l'intestin terminal dont la prévalence varie de $4,4 \%$ à $86 \%$, provoquant une gêne considérable chez ceux qui en souffrent (Johanson et Sonnenberg, 1990 ; Madoff et Fleshman, 2004). Cette maladie affecte aussi bien l'homme que la femme ; mais, à symptômes égaux, les hommes s'en plaignent de façon plus précoce (Haas et al., 1984). L'affection hémorroïdaire apparaît le plus souvent après 30 ans et son incidence dans les pays à haut niveau de vie est estimée à $50 \%$ de la population âgée de 50 ans et plus (Golligher, 1984). La maladie hémorroïdaire motive l'hospitalisation annuelle de 13 sujets par million d'habitants aux États-Unis. En France, les données d'hospitalisation ont permis d'estimer que 82,9 séjours pour actes chirurgicaux en rapport avec la maladie hémorroïdaire ont été réalisés pour 100000 personnes (Johanson et Sonnenberg, 1990 ; Senéjoux, 2010). Au Cameroun, la fréquence de l'affection hémorroïdaire est estimée à $40,83 \%$ des pathologies digestives basses 
(Ankouane et al., 2013). La liste des présumés responsables de cette maladie est longue et parfois fantaisiste (troubles du transit, sédentarité, épices, épisodes de la vie génitale chez la femme, alcool, pratique de certains sports, voyages, etc.). Toutefois, l'alimentation non équilibrée, particulièrement les régimes riches en résidus et pauvres en fibres, la constipation, la position assise prolongée et les épisodes de la vie génitale féminine sont les facteurs les plus incriminés. La pathologie hémorroïdaire revêt des tableaux cliniques très divers. II s'agit en l'occurrence des rectorragies, prurits, prolapsus hémorroïdaires, thromboses hémorroïdaires entrainant des douleurs permanentes et exacerbées, incontinences, marisques ainsi que des cancers (Faucheron, 2005; Boureima, 2006). Le traitement de cette maladie passe par l'adoption des règles hygiéno-diététiques (l'éviction des épices, de l'alcool, du tabac et du café), l'utilisation des topiques (crème, suppositoires), des veinotoniques (diosmine, troxérutine, rutoside) ainsi que des antalgiques et des anti-inflammatoires non stéroïdiens (Senéjoux, 2010). Les méthodes endoscopiques tels les injections sclérosantes, ligatures élastiques de la base d'un paquet hémorroïdaire, la cryothérapie n'enlèvent pas totalement les hémorroïdes et leur efficacité diminue au cours du temps (Senéjoux, 2010 ; Higuero, 2014). Les traitements chirurgicaux quant à eux présentent des résultats satisfaisants mais demeurent très onéreux et occasionnent parfois des effets secondaires incommodes (Lehur et al., 2001 ; Shao et Li, 2008). Les plantes ont fourni des médicaments très efficaces tels que la morphine, l'atropine, l'hyoscyamine, la belladone et bien d'autres (Bézanger-Beauquesne et al., 1975 ; Mpondo et al., 2012 ; Yinyang et al., 2014). Aujourd'hui encore, de nombreuses activités biologiques sont reconnues aux plantes médicinales et sont particulièrement attribuées à leur grande richesse en composés phénoliques (Biaye, 2002 ; Sereme et al., 2008 ; Mpondo et al., 2012 ; Kabran et al., 2014 ; Ngene et al., 2015). De nombreuses plantes ont d'ailleurs montré des effets prononcés contre les affections hémorroïdaires et dont l'usage est reconnu par l'OMS. II s'agit de l'Hamamélis dont les feuilles et l'écorce renferment 8 à $12 \%$ de tanins et de flavonoïdes ; du Ginkgo, du Millepertuis et du Marron d'Inde qui sont riches en ruscogénine et en tanins catéchiques. Les effets veinotoniques, analgésiques, antiinflammatoires et hémostatiques qui sont reconnus à ces plantes sont attribués à ces composés phénoliques (Dupuis, 2013). Les plantes, éléments vitaux de la diversité biologique servent essentiellement au bien être humain (Adjanohoun et al., 2000). En Afrique, près de $80 \%$ de la population ont recourt à la médecine traditionnelle pour se soigner exploitant ainsi les principes actifs des plantes (Biyiti et al., 2004 ; Dibong et al., 2011). De plus, ces plantes constituent des ressources inestimables pour l'industrie pharmaceutique et leurs meilleures exploitation passe par des enquêtes ethnobotaniques qui d'avantage permettent de dresser une liste non exhaustive d'espèces végétales utilisées en médecine traditionnelle par les populations (Awono et al., 2009 ; Dibong et al., 2011 ; Betti et al., 2013 ; Yinyang et al., 2014 ; Ngene et al., 2015). La douleur et la gêne dues aux hémorroïdes sont invalidantes et peuvent entraîner un absentéisme répétitif chez les personnes qui en souffrent et baisser le rendement dans de nombreux secteurs d'activités (Boureima, 2006). L'incapacité de la médecine occidentale à apporter des solutions efficaces face à la maladie hémorroïdaire, le coût élevé des pratiques modernes, les effets secondaires indésirables qui y sont rattachés ainsi que le manque d'infrastructures sanitaire à la pointe poussent les populations à recourir à la médecine traditionnelle (Champigneulle et al., 1989 ; Shao et Li, 2008). De nombreux travaux en Afrique ont permis de recenser un grand éventail de plantes médicinales contre les affections hémorroïdaires et quelques investigations ont été menées afin de justifier l'utilisation de quelques-unes d'entre elles dans le traitement de cette maladie (Dongmo et al., 2007 ; Fézan et al., 2008 ; Gnahoue et al., 2008 ; Mpondo et al., 2012 ; Séguéna et 
al., 2013 ; llumbe et al., 2014 ; Yinyang et al., 2014 ; Ngene et al., 2015). Cependant, malgré les connaissances ethnobotaniques des plantes médicinales, les études sur les plantes anti hémorroïdaires restent embryonnaires et éparses au Cameroun. Le présent travail exploite les savoirs et savoirs faires des herboristes des marchés de vente des plantes médicinales et les détenteurs de connaissances ancestrales des villages du Centre et du Littoral du Cameroun. II a pour

\section{MATERIEL ET METHODES}

Site d'étude : Les zones d'étude sont situées dans les Régions du Centre et du Littoral. La Région du Centre, au centre du pays couvre une superficie de $68926 \mathrm{~km}^{2}$ et abrite plus de 2501200 habitants. Elle appartient au plateau sudcamerounais constitué dans l'ensemble de collines dont l'altitude moyenne est de $600 \mathrm{~m}$. Cette région offre toutes les caractéristiques du climat équatorial typique, avec alternance de deux saisons sèches (mi-novembre à début mars; mijuin à fin août) et de deux saisons des pluies (début mars à mi-juin ; fin août à mi novembre). Sa végétation, caractéristique du climat tropical humide est la forêt dense. Cette dernière recouvre l'ensemble de la Région mais elle est parsemée par endroits de savanes dans laquelle subsistent des lambeaux de forêt. Dans le domaine agricole, la région est favorable aux cultures de rente (cacao, banane, café), aux cultures vivrières (manioc, maïs, arachides et autres) et aux cultures maraîchères (tomate, piment, pastèque et autres). Yaoundé surnommée ville aux sept collines (latitude $3^{\circ} 87^{\prime} \mathrm{N}$; longitude $11^{\circ} 52^{\prime} \mathrm{E}$; altitude $733 \mathrm{~m}$ ) est le chef lieu de la Région du Centre. Yaoundé est la capitale politique du Cameroun et les différents quartiers, disséminés de manière anarchique, laissent une place importante à la végétation dans les bas fonds. Une étude effectuée par le programme ECOFAC (programme de conservation et d'utilisation rationnelle des écosystèmes tropicaux dans l'Afrique Centrale) a identifié Yaoundé comme le principal centre de commerce des produits forestiers non ligneux au Cameroun (Debroux et Dethier, 1993 ; Betti et al, 2002). La Région du Littoral est un ensemble d'écosystèmes où se côtoient les forêts denses humides sempervirentes notamment la forêt dense humide sempervirente littorale entre 0 et $100 \mathrm{~m}$ objectif principal de valoriser la médecine à base des plantes dans le traitement des pathologies hémorroïdaires. Spécifiquement il s'agira de (1) Recenser les informations ethnobotaniques concernant les différentes recettes couramment employées dans le traitement des affections hémorroïdaires ; (2) Caractériser les plantes entrant dans la préparation de ces recettes ; (3) Rechercher les principes actifs de ces plantes.

d'altitude et la forêt dense humide biafréenne entre 100 et $500 \mathrm{~m}$ d'altitude (Letouzey, 1985), la mangrove, les marécages, la végétation ripicole, les agro écosystèmes et la végétation plus ou moins anthropisée. Son climat est de type équatorial avec en général deux saisons, une saison sèche qui dure trois mois et une saison des pluies qui dure neuf mois avec le maximum de précipitations de juillet à octobre (Din et al., 2002). $\mathrm{Ce}$ climat favorise le développement d'une diversité de PFNLs ; cependant ces écosystèmes terrestres et hydromorphes subissent d'énormes pressions anthropiques telles que la pollution surtout en milieu urbain et la déforestation en milieu périurbain (Priso et al., 2011). Douala (latitude, $03^{\circ} 40^{\prime}-04^{\circ} 11^{\prime} \mathrm{N}$; longitude, $09^{\circ} 16^{\prime}-09^{\circ}$ $52^{\prime} \mathrm{E}$; altitude, $13 \mathrm{~m}$ ) est le chef-lieu de la Région du Littoral et compte quatre départements : le Moungo, la Sanaga Maritime, le Nkam et le Wouri. Douala a environ 2,5 millions d'habitants et la croissance démographique n'est pas toujours suivie d'une augmentation des ressources économiques (Priso et al., 2011). Ville cosmopolite, Douala s'est imposée comme capitale économique du pays par son port qui a permis le développement de près de $80 \%$ de l'activité industrielle du Cameroun. Yabassi (latitude $4^{\circ} 37^{\prime}-4^{\circ} 54^{\prime} \mathrm{N}$; longitude $9^{\circ} 87^{\prime}-10^{\circ} 12^{\prime} \mathrm{E}$ ; altitude moyenne 15 à $20 \mathrm{~m}$ correspondant à la valée du Nkam), se trouve dans le département du Nkam, dans la Région du Littoral. La population du canton Yabassi est estimée à près de 10668 habitants. Les principales activités socioéconomiques sont l'agriculture, la pêche, la chasse et l'élevage. Yabassi est une zone forestière du point de vue floristique et l'exploitation des PFNLs apparait comme une activité alternative génératrice de revenus (SNGDESEAC, 2007). 


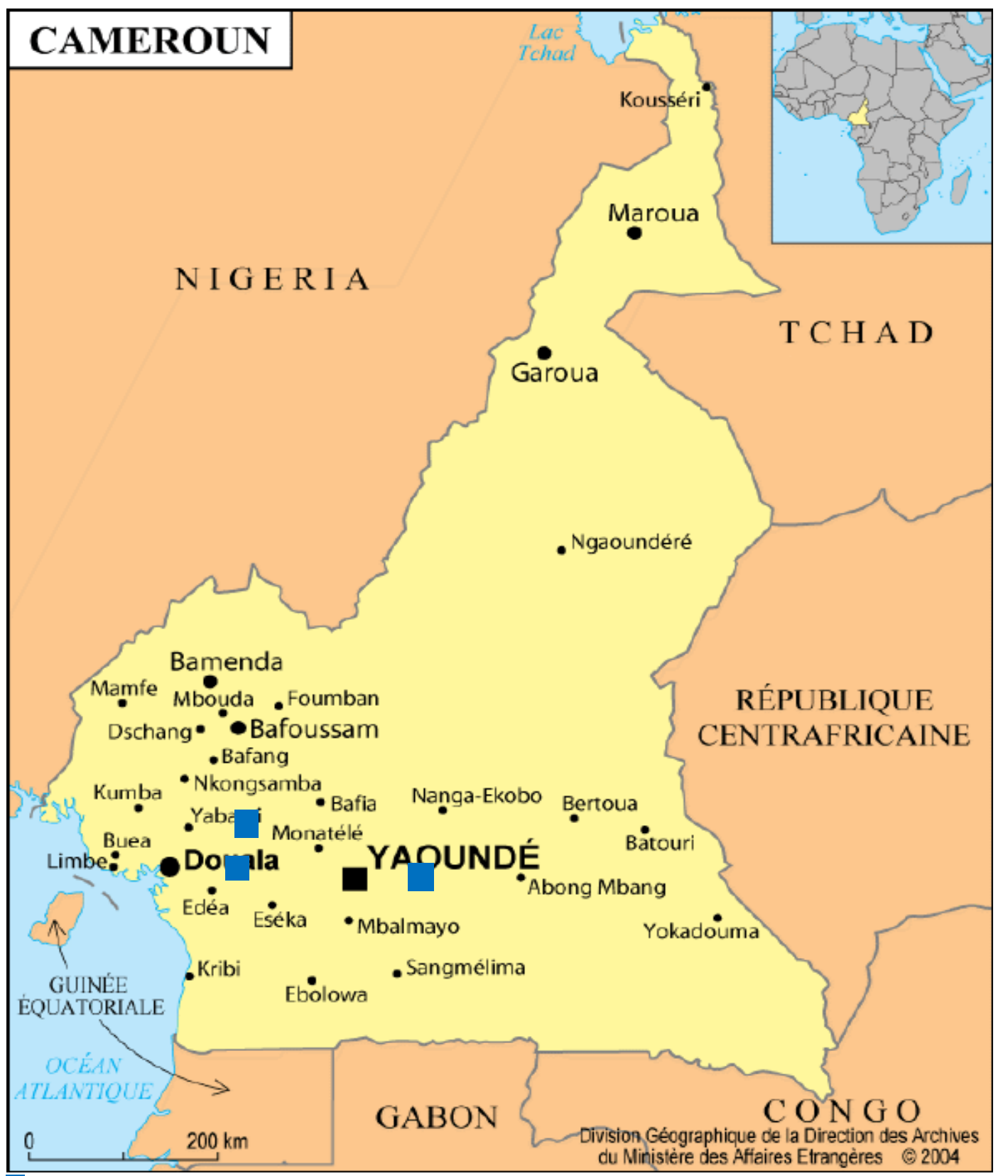

Zones d'étude

Figure 1 : Carte de localisation des Sites étudiés.

Source : Division Géographique de la Direction des Archives du Ministère des Affaires Étrangères, 2004.

Le choix des villages s'est fait sur la base d'enquêtes préalables auprès des personnes autrefois atteintes d'affections hémorroïdaires et ayant trouvé la guérison par le biais de la médecine traditionnelle. Celui des marchés portait sur le fait que le Marché Mokolo et le Marché des Chèvres sont selon Betti (2002) et Priso et al. (2010), les plus grands des deux métropoles en termes de vente des plantes médicinales.

Méthodes d'enquêtes : Des séries d'enquêtes ethnobotaniques ont été réalisées dans deux villages et un grand marché des Régions du Centre et du Littoral dans le but de recenser les différentes recettes utilisées dans le traitement des affections hémorroïdaires. Ces enquêtes semi structurées ont été conduites durant la période allant d'avril à juillet 2015 sur la base d'une fiche d'enquête. Les grandes lignes de l'interview concernaient les informations sur les plantes entrant dans les recettes utilisées contre les hémorroïdes, le mode de préparation de ces recettes, leurs modes d'administration ainsi que les 
autres maladies traitées par ces plantes. En outre, le coût et la durée des traitements ont également été relevés. L'approche des populations était basée sur le dialogue en langues française et locales (Douala, Ewondo et Yabassi). L'accès et l'entretien dans les villages ont été facilités par un guide autochtone du village qui traduisait également la langue locale en français. Quarantedeux personnes dont 8 dans les villages du Centre, 6 au Marché Mekolo et 21 dans les villages du Littoral, 7 au Marché des Chèvres ont été interviewés. Ces interviewés comptent 24 femmes et 18 hommes parmi lesquels 8 tradithérapeutes reconnus, 5 infirmières, 20 herboristes vendeurs de plantes médicinales dans les marchés, 9 guérisseurs détenteurs de savoirs ancestraux. Ils étaient tous adultes âgés d'au moins 35 ans et avaient tous une expérience d'au moins 10 ans dans l'exercice de leur fonction. Tous ont pu reconnaitre la maladie et en décrire les causes et les signes cliniques. Comme il s'agissait des herboristes vendeurs, tradithérapeutes et guérisseurs, leur approche et leur contribution ont été facilitées par l'achat d'une bouteille de bière que chacun consommait pendant l'entretien ainsi qu'une somme de $1000 \mathrm{~F}$ CFA qu'il recevait à la fin de cet entretien. Cette somme couvrait les droits d'acquisition de savoir, l'achat des échantillons d'organes pour les herboristes vendeurs et le droit de machette pour l'entrée en brousse des tradithérapeutes et guérisseurs.

Méthodes d'identification : La récolte d'échantillons dans les villages s'est faite in situ grâce aux détenteurs de savoirs et les tradithérapeutes qui se rendaient disponibles pour entrer dans la brousse. Dans les marchés par contre, l'obtention des échantillons de plantes se faisait par simple achat des organes utilisés dans la recette indiquée. Ces échantillons ont été conservés dans un herbier témoin selon les techniques et méthodes de Schnell (1960). L'identification s'est faite par simple comparaison des noms vernaculaires et/ou commerciaux obtenus auprès des enquêtés, des échantillons de l'herbier témoin avec les données disponibles dans la littérature, des sites web (PROTAU4. ORG) et le matériel de référence disponible au Laboratoire de Biologie et Physiologie des Organismes Végétaux, de l'Université de Douala. Elle a été approuvée par les botanistes du Département de Biologie des Organismes Végétaux de la Faculté des Sciences, de l'Université de Douala. La florule des PFNLs collectés a été caractérisée par les types de distribution phytogéographiques, les types morphologiques, les types de biotopes, ainsi que les types et mode de dissémination des diaspores (Mullenders, 1954 ; Aubreville, 1962 ; Evrard, 1968 ; Letouzey 1985 ; White, 1986). La recherche des principes actifs des plantes anti hémorroïdaires recensées a été rendue possible grâce à la documentation disponible dans les revues ethnopharmacologiques, phytochimiques et botaniques.

Méthodes de saisie et d'analyses des données: Le dépouillement des données a commencé par la saisie des différentes recettes et leurs caractéristiques par site et par informateur dans le tableur Excel Version 2013. S'en est suivi l'encodage des informateurs et enfin le traitement statistique des différentes données. Un dendrogramme montrant la similarité d'utilisation des plantes anti hémorroïdaires par site et par informateur a été établi à partir du coefficient de similarité de Jaccard en utilisant le logiciel XIStat. Le calcul de ce coefficient est basé sur la simple présence ou absence d'une espèce dans une recette citée par un informateur d'un site donné. IL permet de quantifier le degré d'association des espèces et le niveau de similitude entre les recettes en tenant compte des plantes qui y sont utilisées (llumbe et al., 2014). La fréquence des espèces dans chaque site a été évaluée. La fréquence absolue d'une espèce $A$ est le nombre de fois où l'espèce $A$ apparaît. Ainsi, la fréquence relative d'une espèce $A$ est la somme des pourcentages du nombre de sites où elle apparaît sur le nombre total de sites (Priso et al., 2010). Afin d'apprécier les accords des informateurs sur l'utilisation des plantes contre les affections hémorroïdaires, un facteur (degré) de consensus d'utilisation ou Informant Consensus Factor (ICF) a été calculé selon la formule suivante

: ICF $=\frac{N a}{N t}$ où $\mathrm{Na}$ est le nombre d'informateurs ayant cité une espèce et $N t$ le nombre total d'informateurs (llumbe et al., 2014). 


\section{RESULTATS}

$\mathrm{Au}$ total, 60 espèces médicinales anti hémorroïdaires ont été recensées parmi lesquelles 45 ligneux et 15 herbacées. Elles sont reparties dans 41 genres appartenant à 41 familles dont la plus représentée est celle des Fabaceae. Elle est suivie de la famille des Euphorbiaceae. Ces espèces rentrent dans la préparation de 42 recettes parmi lesquelles huit (08) sont des associations de deux plantes, deux utilisent uniquement une (01) seule plante et le reste sont des associations de plus de deux (02) plantes (Tableau 1).

Similitude des connaissances des informateurs : Le dendrogramme permet de

savoir s'il y a rapprochement des connaissances des informations d'un même site ou alors d'un site à l'autre sur les recettes anti hémorroïdaires. L'analyse de ce dendrogramme révèle le caractère disparate des connaissances relatives à l'utilisation des plantes médicinales dans le traitement des maladies hémorroïdaires. Toutefois, $100 \%$ de similitudes s'observent entre les informateurs E9VLN et E2VLN ; entre E6VLN et E11VLN ; E4VLB, E1VLB et E9VLB, entre E3VCM et E2VCM et enfin entre les informateurs E7VLN et E6VLB (Figure 2). Seules les enquêtés d'un même village ont $100 \%$ de similitudes exceptés les enquêtés E7 du village Ndogbélé et E6 du village Bomono.

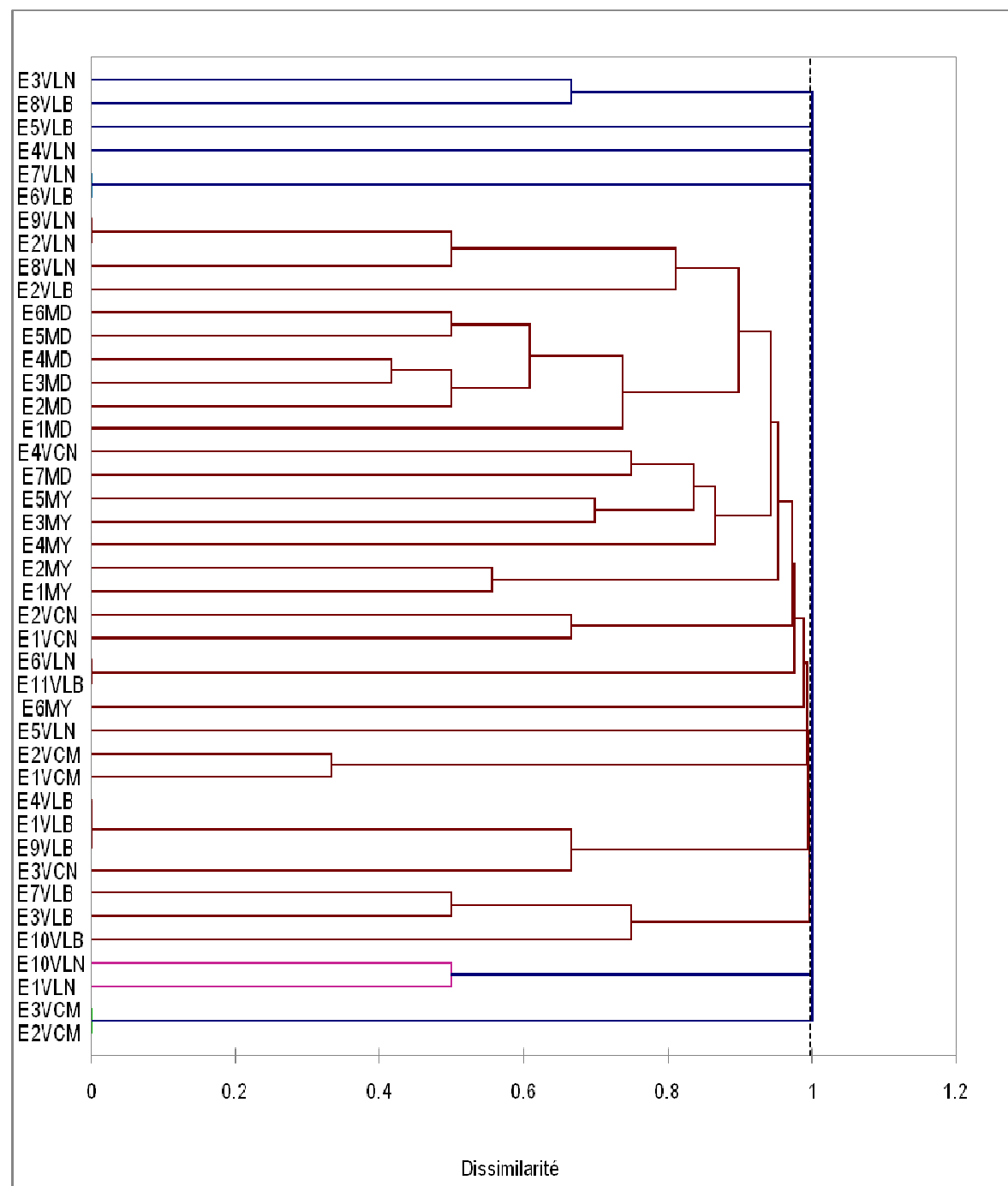

Figure 2: Dendrogramme présentant les similitudes d'emplois des plantes anti-hémorroïdaire entre les spécialistes des marchés et des villages du Centre et du Littoral.

$E=E n q u e ̂ t e ́, \quad M=$ Marché, $\quad C=$ Centre, $\quad L=L i t t o r a l, \quad V=V i l l a g e, \quad N=$ Ndogbélé/Nkolmefou, $B=B o m o n o, \quad M=M e s s a s s i$, $Y=Y$ aoundé, $D=$ Douala.

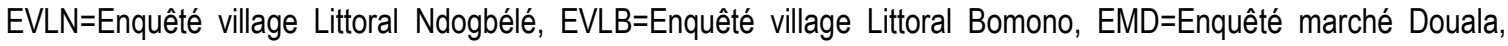
EVCN=Enquêté village Centre Nkolmefou, EVCM=Enquêté village Centre Messassi, EMY=Enquêté marché Yaoundé. 
Tableau 1: Informations relatives aux recettes anti hémorroïdaires et aux plantes entrant dans la préparation de ces recettes.

\begin{tabular}{|c|c|c|c|c|c|}
\hline $\begin{array}{l}\text { Noms scientifiques/ } \\
\text { vernaculaires } \\
\text { (Familles) }\end{array}$ & Maladies soignées & $\begin{array}{l}\text { Organes } \\
\text { utilisés }\end{array}$ & $\begin{array}{l}\text { Plantes et/ou éléments associés et autres } \\
\text { précisions }\end{array}$ & $\begin{array}{l}\text { Modes de } \\
\text { préparation }\end{array}$ & $\begin{array}{l}\text { Modes } \\
\text { d'administration }\end{array}$ \\
\hline $\begin{array}{l}\text { Acmella caulirhza/ Yeux } \\
\text { de la poule } \\
\text { (Asteraceae) }\end{array}$ & & $\begin{array}{l}\text { Plante } \\
\text { entière }\end{array}$ & $\begin{array}{l}\text { Euphorbia hirta+Piper umbellatum+Dissotis } \\
\text { rotundifolia + Psidium guajava } \\
\text { Desmodium adscendens +Elaeis guineensis }\end{array}$ & $\begin{array}{l}\text { Trituration } \\
\text { Calcination+ Huile } \\
\text { végétale }\end{array}$ & $\begin{array}{l}\text { Boire 3fois/ } \\
\text { jour } \\
\text { Suppositoire } \\
\text { 2fois/jour }\end{array}$ \\
\hline $\begin{array}{l}\text { Adansonia digitatal } \\
\text { Baobab } \\
\text { (Bombacaceae) }\end{array}$ & Trompes bouchées & Écorces & $\begin{array}{l}\text { Mangifera indica +Pycnanthus angolensis } \\
\text { +Antrocaryon klaineanum + Cylicodiscus } \\
\text { gabonensis }\end{array}$ & Décoction à l'eau & $\begin{array}{l}\text { Boire 2fois/jour + Bain } \\
\text { de siège 2fois/jour }\end{array}$ \\
\hline $\begin{array}{l}\text { Afromomum danielliil } \\
\text { Mbongo (Zingiberaceae) }\end{array}$ & & $\begin{array}{l}\text { Graines } \\
\text { du fruit }\end{array}$ & Guilbourtia tesmannii & Décoction à l'eau & $\begin{array}{l}\text { Boire+ Bain de siège } \\
\text { 2fois/jour }\end{array}$ \\
\hline $\begin{array}{l}\text { Ageratum conyzoïdes/ } \\
\text { Rois des herbes } \\
\text { (Asteraceae) }\end{array}$ & Règles douloureuses & $\begin{array}{l}\text { Plante } \\
\text { entière }\end{array}$ & $\begin{array}{l}\text { Capsicum frutescens + } \\
\text { Ocimum gratissimum }\end{array}$ & Écraser & $\begin{array}{l}\text { Suppositoire } \\
\text { 1fois/jour }\end{array}$ \\
\hline $\begin{array}{l}\text { Alchornea cordifolial } \\
\text { Aboe } \\
\text { (Euphorbiaceae) }\end{array}$ & Anémie & Feuilles & Capsicum frutescens + Elaeis guineensis & $\begin{array}{l}\text { Écraser + } \\
\text { Huile végétale }\end{array}$ & $\begin{array}{l}\text { Suppositoire } \\
\text { 1fois/jour }\end{array}$ \\
\hline $\begin{array}{l}\text { Alstonia booneilEkuk } \\
\text { (Apocynaceae) }\end{array}$ & $\begin{array}{l}\text { Diabète, Typhoïde, } \\
\text { Paludisme, Jaunisse }\end{array}$ & Écorces & Lorsque les Hémorroïdes font mal au ventre & Macération à l'eau & Boire 3fois/jour \\
\hline $\begin{array}{l}\text { Anchomanes difformis/ } \\
\text { Dindela bedimo } \\
\text { (Araceae) }\end{array}$ & Abcès, Douleurs & Rhizomes & Laportea ovalifolia + Elaeis guineensis & $\begin{array}{l}\text { Râper+ } \\
\text { Huile } \\
\text { végétale }\end{array}$ & $\begin{array}{l}\text { Suppositoire } \\
\text { 2fois/jour }\end{array}$ \\
\hline $\begin{array}{l}\text { Annoduim maniil Ebom } \\
\text { afan } \\
\text { (Annonaceae) }\end{array}$ & Facilite la lactation & Écorces & $\begin{array}{l}\text { Cola chlamydantha + Cylicodiscus gabonensis } \\
\text { +Baillonella toxisperma }\end{array}$ & Décoction à l'eau & $\begin{array}{l}\text { Boire 2fois/jour + Bain } \\
\text { de siège 1fois/jour }\end{array}$ \\
\hline $\begin{array}{l}\text { Annona senegalensis/ } \\
\text { Ebom Nlong } \\
\text { (Annonaceae) }\end{array}$ & $\begin{array}{l}\text { IST, Faiblesse } \\
\text { sexuelle }\end{array}$ & Écorces & $\begin{array}{l}\text { Mangifera indica +Pycnanthus angolensis } \\
+ \text { Antrocaryon klaineanum + Alstonia boonei }\end{array}$ & Décoction à l'eau & $\begin{array}{l}\text { Boire+ Bain de siège } \\
\text { 2fois/jour }\end{array}$ \\
\hline $\begin{array}{l}\text { Antrocaryon } \\
\text { klaineanum/ Angongui } \\
\text { (Anacardiaceae) }\end{array}$ & $\begin{array}{l}\text { IST, Règles } \\
\text { douloureuses, } \\
\text { Mal de ventre }\end{array}$ & Écorces & $\begin{array}{l}\text { Entandrophragma cylindricum +Morinda lucida } \\
\text { +Lophira alata +Irvingia gabonensis } \\
\text { +Baillonella toxisperma }\end{array}$ & Décoction à l'eau & $\begin{array}{l}\text { Boire 2fois/jour }+ \\
\text { Bain de siège 1fois/jour }\end{array}$ \\
\hline $\begin{array}{l}\text { Baillonella toxispermal } \\
\text { Adjap }\end{array}$ & $\begin{array}{l}\text { Mal de dos, Mal de } \\
\text { reins, Inflammations }\end{array}$ & Écorces & $\begin{array}{l}\text { Guilbourtia tesmannii+Mammea africana + } \\
\text { Canarium schweinfurthii + Entandrophragma }\end{array}$ & Décoction à l'eau & $\begin{array}{l}\text { Boire 2fois/jour + Bain } \\
\text { de siège 1fois/jour }\end{array}$ \\
\hline
\end{tabular}


Dibong et al. J. Appl. Biosci. 2015 Ethnobotanique des plantes médicinales anti hémorroïdaires des marchés et villages du Centre et du Littoral Cameroun

\begin{tabular}{|c|c|c|c|c|c|}
\hline (Sapotaceae) & & & candollei + Spathodea campanulata & & \\
\hline $\begin{array}{l}\text { Bridelia micranthal } \\
\text { Ewolot } \\
\text { (Euphorbiaceae) }\end{array}$ & & Écorces & $\begin{array}{l}\text { Baillonella toxisperma + Cylicodiscus } \\
\text { gabonensis +Ricinodendron heudelotii }\end{array}$ & Décoction à l'eau & $\begin{array}{l}\text { Boire 2fois/jour } \\
+ \text { Bain de siège } \\
\text { 1fois/jour }\end{array}$ \\
\hline $\begin{array}{l}\text { Canarium schweinfurthiil } \\
\text { Aiele } \\
\text { (Burseraceae) }\end{array}$ & Anémie, IST & Écorces & $\begin{array}{l}\text { Spathodea campanulata + Mammea africana+ } \\
\text { Entandrophragma candollei }\end{array}$ & Décoction à l'eau & $\begin{array}{l}\text { Boire 2fois/jour } \\
+ \text { Bain de siège } \\
\text { 1fois/jour }\end{array}$ \\
\hline $\begin{array}{l}\text { Capsicum frutescens/ } \\
\text { Ondondo beloe } \\
\text { (Solanaceae) }\end{array}$ & & Fruits & $\begin{array}{l}\text { Piper umbellatum+ Ocimum gratissimum + } \\
\text { Alchornea cordifolia+ Elaeis guineensis }\end{array}$ & $\begin{array}{l}\text { Écraser+ Huile } \\
\text { végétale }\end{array}$ & Suppositoire le soir \\
\hline $\begin{array}{l}\text { Citrus lemon/ Citron } \\
\text { (Rutaceae) }\end{array}$ & Mal de ventre, Fatigue & Fruits & Eremomastax speciosa & Fermenter à l'eau & Boire 2fois/jour \\
\hline $\begin{array}{l}\text { Cola chlamydanthal } \\
\text { Epok } \\
\text { (Sterculiaceae) }\end{array}$ & $\begin{array}{l}\text { Fortifie la femme } \\
\text { enceinte, Anémie }\end{array}$ & Écorces & $\begin{array}{l}\text { Cylicodiscus gabonensis + Baillonella } \\
\text { toxisperma+ } \\
\text { Hylodendron gabunense +Musanga } \\
\text { cecropioides }\end{array}$ & Décoction à l'eau & $\begin{array}{l}\text { Boire 2fois/jour } \\
+ \text { Bain de siège } \\
\text { 1fois/jour }\end{array}$ \\
\hline $\begin{array}{l}\text { Costus aferl Canne des } \\
\text { jumeaux } \\
\text { (Costaceae) }\end{array}$ & $\begin{array}{l}\text { Typhoïde, Diarrhée, } \\
\text { Paludisme }\end{array}$ & $\begin{array}{l}\text { Tige } \\
\text { feuillée }\end{array}$ & $\begin{array}{l}\text { Phragmantera capitata } \\
\text { Emilia coccinea + Elaeis guineensis }\end{array}$ & $\begin{array}{l}\text { Décoction à l'eau } \\
\text { Chauffer+ } \\
\text { écraser+ Huile } \\
\text { végétale }\end{array}$ & $\begin{array}{l}\text { Boire 2fois/jour+ Bain de } \\
\text { siège 1fois/jour } \\
\text { Suppositoire 1fois/jour }\end{array}$ \\
\hline $\begin{array}{l}\text { Croton oligandrus/ Bin } \\
\text { (Euphorbiaceae) }\end{array}$ & kystes, purifie le sang & Écorces & $\begin{array}{l}\text { Mangifera indica +Irvingia gabonensis } \\
+ \text { Lophira alata }\end{array}$ & Décoction à l'eau & Boire 2fois/jour \\
\hline $\begin{array}{l}\text { Cyathula prostratal } \\
\text { Kolokossi } \\
\text { (Amarantheceae) }\end{array}$ & Diarrhée & Feuilles & $\begin{array}{l}\text { Ziziphus lotus + Laportea ovalifolia + Piper } \\
\text { umbellatum }\end{array}$ & Préparer le mets & Manger autant de fois \\
\hline $\begin{array}{l}\text { Cylicodiscus } \\
\text { gabonensis/ Adoum } \\
\text { (Mimosaceae) }\end{array}$ & $\begin{array}{l}\text { Démangeaisons, } \\
\text { Nettoyage, } \\
\text { Antibiotique }\end{array}$ & Écorces & $\begin{array}{l}\text { Mangifera indica }+ \text { Pycnanthus angolensis }+ \\
\text { Adansonia digitata }+ \text { Antrocaryon klaineanum }\end{array}$ & Décoction à l'eau & $\begin{array}{l}\text { Boire 2fois/jour } \\
+ \text { Bain de siège } \\
\text { 1fois/jour }\end{array}$ \\
\hline $\begin{array}{l}\text { Cymbopogon citratus/ } \\
\text { Citronnelle (Poaceae) }\end{array}$ & Pieds enflés & Feuilles & Senna alata (Lutte contre la fatigue) & Décoction à l'eau & $\begin{array}{l}\text { Ajouter } 1 \text { sucre Boire } \\
1 \text { fois/jour }\end{array}$ \\
\hline $\begin{array}{l}\text { Desmodium } \\
\text { adscendens/ Arachide } \\
\text { fantôme } \\
\text { (Fabaceae) }\end{array}$ & & $\begin{array}{l}\text { Plante } \\
\text { entière }\end{array}$ & $\begin{array}{l}\text { Euphorbia hirta + Piper umbellatum+ Sida } \\
\text { acuta +Dissotis rotundifolia }\end{array}$ & $\begin{array}{l}\text { Calcination+ Huile } \\
\text { végétale }\end{array}$ & Suppositoire 2fois/jour \\
\hline Dissotis rotundifolia/ Le & Règles douloureuses & Plante & Piper umbellatum+ Sida acuta + Elaeis & Calcination+ & Suppositoire 2fois/jour \\
\hline
\end{tabular}


Dibong et al. J. Appl. Biosci. 2015 Ethnobotanique des plantes médicinales anti hémorroïdaires des marchés et villages du Centre et du Littoral Cameroun

\begin{tabular}{|c|c|c|c|c|c|}
\hline Salé (Melastomataceae) & & entière & guineensis & Huile végétale & \\
\hline $\begin{array}{l}\text { Elaeis guineensis/ Huile } \\
\text { rouge/ Manyanga } \\
\text { (Arecaceae) }\end{array}$ & & $\begin{array}{l}\text { Huile des } \\
\text { noix }\end{array}$ & $\begin{array}{l}\text { Piper umbellatum+ Sida acuta +Dissotis } \\
\text { rotundifolia + Anchomanes difformis }\end{array}$ & Calcination & Suppositoire 2fois/jour \\
\hline $\begin{array}{l}\text { Emilia coccinea/ Oreille } \\
\text { chien (Asteraceae) }\end{array}$ & Otite & Feuilles & Costus afer + Elaeis guineensis & Chauffer+ écraser & Lécher le mélange \\
\hline $\begin{array}{l}\text { Entandrophragma } \\
\text { candolleil Mevenga } \\
\text { (Meliaceae) }\end{array}$ & Antibiotique & Écorces & $\begin{array}{l}\text { Morinda lucida + Baillonella toxisperma+ } \\
\text { Hylodendron gabunense + Canarium } \\
\text { schweinfurthii }\end{array}$ & Décoction à l'eau & $\begin{array}{l}\text { Boire 2fois/jour } \\
+ \text { Bain de siège } \\
\text { 1fois/jour }\end{array}$ \\
\hline $\begin{array}{l}\text { Entandrophragma } \\
\text { cylindricum/ Koundjock } \\
\text { (Meliaceae) }\end{array}$ & Anti inflammatoire & Écorces & $\begin{array}{l}\text { Petersianthus macrocarpus + Lophira alata+ } \\
\text { Morinda lucida + Baillonella toxisperma }\end{array}$ & Décoction à l'eau & Bain de siège 1fois/jour \\
\hline $\begin{array}{l}\text { Entandrophragma utile/ } \\
\text { Asseng Assié } \\
\text { (Meliaceae) }\end{array}$ & Antibiotique, IST & Écorces & $\begin{array}{l}\text { Croton oligandrus + Lophira alata+ Morinda } \\
\text { lucida + Pycnanthus angolensis }\end{array}$ & Décoction à l'eau & $\begin{array}{l}\text { Boire 2fois/jour+ Bain de } \\
\text { siège 1fois/jour }\end{array}$ \\
\hline $\begin{array}{l}\text { Eremomastax speciosal } \\
\text { Rouge d'un coté } \\
\text { (Acanthaceae) }\end{array}$ & Erythèmes fessiers & Feuilles & Citrus lemon (2 - 4 gouttes au plus) & Fermenter à l'eau & $\begin{array}{l}\text { Boire 2fois/jour + } \\
\text { Suppositoire 1fois/jour }\end{array}$ \\
\hline $\begin{array}{l}\text { Erythrophleum } \\
\text { suaveolens/ Elon } \\
\text { (Ceasalpiniaceae) }\end{array}$ & $\begin{array}{l}\text { Panaris, Anti } \\
\text { inflammatoire }\end{array}$ & Écorces & & Décoction à l'eau & $\begin{array}{l}\text { Bain de siège 3f/j (Pas } \\
\text { boire) }\end{array}$ \\
\hline $\begin{array}{l}\text { Euphorbia hirtal Lait de } \\
\text { bébé (Euphorbiaceae) }\end{array}$ & Infection pulmonaire, & $\begin{array}{l}\text { Plante } \\
\text { entière }\end{array}$ & $\begin{array}{l}\text { Acmella caulirhza + Sida acuta + Piper } \\
\text { umbellatum }\end{array}$ & Triturer + eau & Boire 2fois/jour \\
\hline $\begin{array}{l}\text { Ficus exasperatal Akol } \\
\text { (Moraceae) }\end{array}$ & Chaude pisse & Feuilles & Pistache & Préparer le mets & Manger autant de fois \\
\hline $\begin{array}{l}\text { Guibourtia tessmanniil } \\
\text { Essingang } \\
\text { (Caesalpiniaceae) }\end{array}$ & $\begin{array}{l}\text { Paludisme, } \\
\text { Antibiotique }\end{array}$ & Écorces & $\begin{array}{l}\text { Afromomum daniellii + Spathodea } \\
\text { campanulata+ Baillonella toxisperma + } \\
\text { Pycnanthus angolensis }\end{array}$ & Décoction à l'eau & $\begin{array}{l}\text { Boire 2fois/jour } \\
+ \text { Bain de siège } \\
\text { 1fois/jour }\end{array}$ \\
\hline $\begin{array}{l}\text { Hylodendron } \\
\text { gabunense/ Sikon } \\
\text { (Euphorbiaceae) }\end{array}$ & $\begin{array}{l}\text { IST, Syphilis, } \\
\text { Chlamydia }\end{array}$ & Écorces & $\begin{array}{l}\text { Cola chlamydantha + Annoduim manii+ } \\
\text { Cylicodiscus gabonensis + Baillonella } \\
\text { toxisperma + Musanga cecropioides }\end{array}$ & Décoction à l'eau & $\begin{array}{l}\text { Boire 2fois/jour } \\
+ \text { Bain de siège } \\
\text { 1fois/jour }\end{array}$ \\
\hline $\begin{array}{l}\text { Irvingia gabonensisl } \\
\text { Andok } \\
\text { (Irvingiaceae) }\end{array}$ & $\begin{array}{l}\text { Vers de femme, } \\
\text { Nettoie la mauvaise } \\
\text { eau }\end{array}$ & Écorces & $\begin{array}{l}\text { Kanwha } \\
\text { Mangifera indica+ Cylicodiscus gabonensis + } \\
\text { Baillonella toxisperma + Antrocaryon }\end{array}$ & Décoction à l'eau & $\begin{array}{l}\text { Boire 2fois/jour } \\
\text { Boire } 2 \text { fois/jour } \\
+ \text { Bain de siège }\end{array}$ \\
\hline
\end{tabular}


Dibong et al. J. Appl. Biosci. 2015 Ethnobotanique des plantes médicinales anti hémorroïdaires des marchés et villages du Centre et du Littoral Cameroun

\begin{tabular}{|c|c|c|c|c|c|}
\hline & & & & & 1fois/jour \\
\hline $\begin{array}{l}\text { Laportea ovalifolial } \\
\text { Tolobandji } \\
\text { (Urticaceae) }\end{array}$ & $\begin{array}{l}\text { Hémiplégie, } \\
\text { inflammation }\end{array}$ & Feuilles & Elaeis guineensis & $\begin{array}{l}\text { Chauffer+ } \\
\text { écraser+ Huile } \\
\text { végétale }\end{array}$ & $\begin{array}{l}\text { Suppositoire } \\
\text { 1fois/jour }\end{array}$ \\
\hline $\begin{array}{l}\text { Lophira alata/ Mangossi } \\
\text { (Ochnaceae) }\end{array}$ & Mal de nerf, Fatigue & Écorces & $\begin{array}{l}\text { Mangifera indica + Irvingia gabonensis + } \\
\text { Antrocaryon klaineanum+ Cylicodiscus }\end{array}$ & Décoction à l'eau & $\begin{array}{l}\text { Boire 2fois/jour } \\
+ \text { Bain de siège } \\
\text { 1fois/jour }\end{array}$ \\
\hline $\begin{array}{l}\text { Mammea africanal } \\
\text { Abodzok } \\
\text { (Clusiaceae) }\end{array}$ & $\begin{array}{l}\text { Gales, } \\
\text { Démangeaisons, } \\
\text { Excès de pertes } \\
\text { blanches }\end{array}$ & Écorces & $\begin{array}{l}\text { Guibourtia tessmannii + Baillonella toxisperm+ } \\
\text { Spathodea campanulata + Pycnanthus } \\
\text { angolensis }\end{array}$ & Décoction à l'eau & $\begin{array}{l}\text { Boire 2fois/jour } \\
+ \text { Bain de siège } \\
\text { 1fois/jour }\end{array}$ \\
\hline $\begin{array}{l}\text { Mangifera indica/ Ndo'o } \\
\text { ntangan } \\
\text { (Anacardiaceae) }\end{array}$ & $\begin{array}{l}\text { Mal de dents, anti } \\
\text { inflammatoire }\end{array}$ & Écorces & $\begin{array}{l}\text { Adansonia digitata+ Antrocaryon klaineanum+ } \\
\text { Cylicodiscus gabonensis }\end{array}$ & Décoction à l'eau & $\begin{array}{l}\text { Boire 2fois/jour } \\
+ \text { Bain de siège } \\
\text { 1fois/jour }\end{array}$ \\
\hline $\begin{array}{l}\text { Morinda lucidal Akeng } \\
\text { (Rubiaceae) }\end{array}$ & $\begin{array}{l}\text { Facilite la digestion, } \\
\text { Constipation }\end{array}$ & Écorces & $\begin{array}{l}\text { Picralima nitida +Baillonella toxisperma } \\
\text { +Antrocaryon klaineanum }\end{array}$ & Décoction à l'eau & $\begin{array}{l}\text { Boire 2fois/jour } \\
+ \text { Bain de siège } \\
\text { 1fois/jour }\end{array}$ \\
\hline $\begin{array}{l}\text { Musa paradisiacal } \\
\text { Ekouan } \\
\text { (Musaceae) }\end{array}$ & & $\begin{array}{l}\text { Rejeton } \\
\text { Fruits }\end{array}$ & $\begin{array}{l}\text { Spathodea campanulata Antrocaryon } \\
\text { klaineanum }\end{array}$ & Décoction à l'eau & $\begin{array}{l}\text { Boire 3fois/jour + Purge } \\
\text { après 3j }\end{array}$ \\
\hline $\begin{array}{l}\text { Musanga cecropioides/ } \\
\text { Asseng } \\
\text { (Cecropiaceae) }\end{array}$ & $\begin{array}{l}\text { Vers de femmes, } \\
\text { Nettoie le ventre }\end{array}$ & Écorces & $\begin{array}{l}\text { Baillonella toxisperma + Cola } \\
\text { chlamydantha+Cylicodiscus gabonensis + } \\
\text { Hylodendron gabunense }\end{array}$ & Décoction à l'eau & $\begin{array}{l}\text { Boire 2fois/jour } \\
+ \text { Bain de siège } \\
\text { 1fois/jour }\end{array}$ \\
\hline $\begin{array}{l}\text { Myrianthus arboreus/ } \\
\text { Ingakouam } \\
\text { (Moraceae) }\end{array}$ & & Écorces & $\begin{array}{l}\text { Trilepisium madagascariense + Bridelia } \\
\text { micrantha + Baillonella toxisperma + } \\
\text { Pachyelasma tessmannii }\end{array}$ & Décoction à l'eau & $\begin{array}{l}\text { Boire 2fois/jour } \\
+ \text { Bain de siège } \\
1 \text { fois/jour + Purge } \\
\text { 1fois/4Se }\end{array}$ \\
\hline $\begin{array}{l}\text { Ocimum gratissimuml } \\
\text { Massep } \\
\text { (Lamiaceae) }\end{array}$ & $\begin{array}{l}\text { Douleurs au bas } \\
\text { ventre, Indigestion }\end{array}$ & Feuilles & $\begin{array}{l}\text { Ageratum conyzoïdes + Capsicum } \\
\text { frutescens+ Elaeis guineensis }\end{array}$ & $\begin{array}{l}\text { Triturer (Seul) } \\
\text { Écraser+ HV }\end{array}$ & $\begin{array}{l}\text { Boire 2fois/jour } \\
\text { Suppositoire } \\
\text { 1fois/jour }\end{array}$ \\
\hline $\begin{array}{l}\text { Pachyelasma } \\
\text { tessmanniil Eyeuk } \\
\text { (Caesalpiniaceae) }\end{array}$ & $\begin{array}{l}\text { Stoppe les } \\
\text { hémorragies }\end{array}$ & Écorces & $\begin{array}{l}\text { Trilepisium madagascariense + Bridelia } \\
\text { micrantha + Baillonnella toxisperma + } \\
\text { Guibourtia tessmannii + Myrianthus arboreus }\end{array}$ & Décoction à l'eau & $\begin{array}{l}\text { Boire 2fois/jour } \\
+ \text { Bain de siège } \\
\text { 1fois/jour }\end{array}$ \\
\hline $\begin{array}{l}\text { Palisota barteril A mia } \\
\text { okowa }\end{array}$ & & Feuilles & & Chauffer et triturer & $\begin{array}{l}\text { Suppositoire tous les } \\
\text { soirs }\end{array}$ \\
\hline
\end{tabular}


Dibong et al. J. Appl. Biosci. 2015 Ethnobotanique des plantes médicinales anti hémorroïdaires des marchés et villages du Centre et du Littoral Cameroun

\begin{tabular}{|c|c|c|c|c|c|}
\hline (Commelinaceae) & & & & & \\
\hline $\begin{array}{l}\text { Petersianthus } \\
\text { macrocarpus/ Abin } \\
\text { (Lecythidaceae) }\end{array}$ & Mal de ventre & Écorces & $\begin{array}{l}\text { Irvingia gabonensis + Pycnanthus angolensis } \\
\text { Lophira alata+ Morinda lucida + Baillonella } \\
\text { toxisperma }\end{array}$ & Décoction à l'eau & $\begin{array}{l}\text { Boire 2fois/jour } \\
+ \text { Bain de siège } \\
\text { 1fois/jour } \\
\end{array}$ \\
\hline $\begin{array}{l}\text { Phragmanthera capitatal } \\
\text { Samacopée } \\
\text { (Lorantaceae) }\end{array}$ & $\begin{array}{l}\text { Démangeaisons, } \\
\text { Hypertension }\end{array}$ & Feuilles & Costus afer & Décoction à l'eau & $\begin{array}{l}\text { Boire 2fois/jour } \\
+ \text { Bain de siège } \\
\text { 1fois/jour }\end{array}$ \\
\hline $\begin{array}{l}\text { Picralima nitida/ Ebam } \\
\text { (Apocynaceae) }\end{array}$ & Paludisme & Écorces & $\begin{array}{l}\text { Baillonella toxisperma + Morinda lucida+ } \\
\text { Antrocaryon klaineanu }\end{array}$ & Décoction à l'eau & $\begin{array}{l}\text { Boire+ Bain de siège } \\
\text { 2fois/jour }\end{array}$ \\
\hline $\begin{array}{l}\text { Piper umbellatum/ Bora } \\
\text { (Piperaceae) }\end{array}$ & Sevrage de l'enfant & $\begin{array}{l}\text { Plante } \\
\text { entière }\end{array}$ & $\begin{array}{l}\text { Capsicum frutescens + } \\
\text { Ocimum gratissimum+ } \\
\text { Ageratum conyzoïdes }\end{array}$ & $\begin{array}{l}\text { Écraser }+ \\
\text { Huile végétale }\end{array}$ & Suppositoire 2fois/jour \\
\hline $\begin{array}{l}\text { Piptadenistrum } \\
\text { africanum/ Atui } \\
\text { (Mimosaceae) }\end{array}$ & Antibiotique, & Écorces & $\begin{array}{l}\text { Baillonella toxisperma+ Mammea africana+ } \\
\text { Pycnanthus angolensis+ E. utile }\end{array}$ & Décoction à l'eau & $\begin{array}{l}\text { Boire+ Bain de siège } \\
\text { 2fois/jour }\end{array}$ \\
\hline $\begin{array}{l}\text { Psidium guajaval Afélé } \\
\text { (Myrtaceae) }\end{array}$ & Typhoïde, dysenterie & Feuilles & $\begin{array}{l}\text { Desmodium adscendens +Elaeis guineensis } \\
+ \text { Acmella caulirhza }\end{array}$ & $\begin{array}{l}\text { Calcination+ } \\
\text { Huile végétale }\end{array}$ & $\begin{array}{l}\text { Suppositoire } \\
\text { 2fois/jour }\end{array}$ \\
\hline $\begin{array}{l}\text { Pycnanthus angolensisl } \\
\text { Eteng } \\
\text { (Myristicacea) }\end{array}$ & $\begin{array}{l}\text { Purifie le lait, } \\
\text { Erythèmes fessiers, } \\
\text { Tonifiant }\end{array}$ & Écorces & $\begin{array}{l}\text { Irvingia gabonensis + } \\
\text { Lophira alata+ Morinda lucida + Baillonella } \\
\text { toxisperma }\end{array}$ & Décoction à l'eau & $\begin{array}{l}\text { Boire } 2 \text { fois/jour + Bain } \\
\text { de siège } 1 \mathrm{fois} / \mathrm{j}\end{array}$ \\
\hline $\begin{array}{l}\text { Ricinodendron } \\
\text { heudelotiil Esesang } \\
\text { (Euphorbiaceae) }\end{array}$ & & Écorces & $\begin{array}{l}\text { Guibourtia tessmannii+ Morinda lucida + } \\
\text { Baillonella toxisperma+Cylicodiscus } \\
\text { gabonensis + Bridelia micrantha }\end{array}$ & Décoction à l'eau & $\begin{array}{l}\text { Boire 2fois/jour + Bain } \\
\text { de siège } 1 \text { fois/jour } \\
\text { Purge après } 3 \text { mois }\end{array}$ \\
\hline $\begin{array}{l}\text { Senna alatal Datrier } \\
\text { (Fabaceae) }\end{array}$ & Dartre, Typhoïde & Feuilles & $\begin{array}{l}\text { Cymbopogon citratus }+1 \text { sucre (En cas de } \\
\text { fatigue) }\end{array}$ & Décoction à l'eau & $\begin{array}{l}\text { Boire 1fois/jour pendant } \\
\text { 4jours }\end{array}$ \\
\hline $\begin{array}{l}\text { Sida acuta/ Shimboulou } \\
\text { (Malvaceae) }\end{array}$ & Nettoyage & Feuilles & $\begin{array}{l}\text { Euphorbia hirta+ Acmella caulirhza + Piper } \\
\text { umbellatum +Dissotis rotundifolia }\end{array}$ & Trituration & Boire 3fois/jour \\
\hline
\end{tabular}




\begin{tabular}{|c|c|c|c|c|c|}
\hline \multicolumn{6}{|l|}{$\begin{array}{l}\text { Dibong et al. } \\
\text { Cameroun }\end{array}$} \\
\hline $\begin{array}{l}\text { Spathodea } \\
\text { campanulatal Evevon } \\
\text { (Bignoniaceae) }\end{array}$ & $\begin{array}{l}\text { Antibiotique, } \\
\text { Inflammation, Facilite } \\
\text { la lactation }\end{array}$ & Écorces & $\begin{array}{l}\text { Baillonella toxisperma+ Musa paradisiaca+ } \\
\text { Mammea africana+ Pycnanthus angolensis+ } \\
\text { Entandrophragma candollei+ Canarium } \\
\text { schweinfurthii }\end{array}$ & $\begin{array}{l}\text { Décoction à l'eau } \\
\text { +Doigt de plantain }\end{array}$ & $\begin{array}{l}\text { Boire 2fois/jour + Bain } \\
\text { de siège 1fois/jour } \\
\text { 1purge après 2jours }\end{array}$ \\
\hline $\begin{array}{l}\text { Trilepisium } \\
\text { madagascariense/ Zolibi } \\
\text { (Moraceae) }\end{array}$ & & Écorces & Myrianthus arboreus & $\begin{array}{l}\text { Décoction à } \\
\text { l'alcool + eau }\end{array}$ & Bain de siège 2fois/jour \\
\hline $\begin{array}{l}\text { Ziziphus lotus/ Jujube } \\
\text { (Rhamnaceae) }\end{array}$ & & Feuilles & $\begin{array}{l}\text { Cyathula prostrata + Laportea ovalifolia +Piper } \\
\text { umbellatum }\end{array}$ & $\begin{array}{l}\text { Découper dans le } \\
\text { mets de pistache } \\
\text { et le préparer }\end{array}$ & $\begin{array}{l}\text { Consommer autant de } \\
\text { fois }\end{array}$ \\
\hline
\end{tabular}


Parmi les espèces recensées pour le traitement des maladies hémorroïdaires, les ligneux sont les plus utilisés $(58 \%)$, suivis des herbes vivaces et annuelles (25\%) (Figure 3).

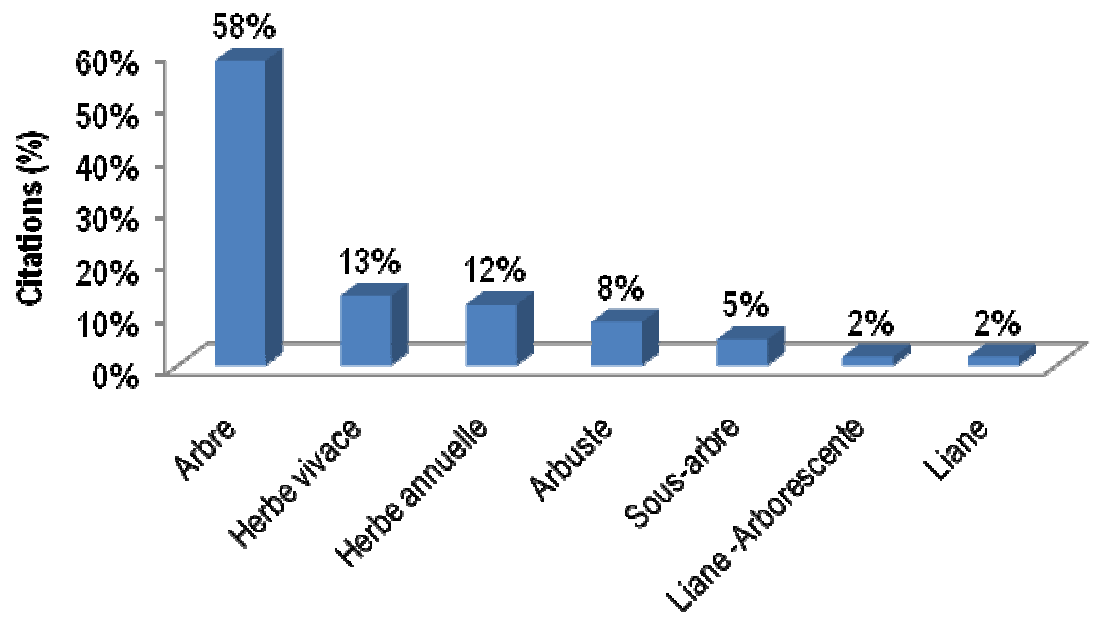

Types morphologiques

Figure 3 : Histogramme (\% en citation) des types morphologiques.

Les proportions de la distribution à distribution régionale (62\%). Parmi ces espèces, phytogéographique des espèces recensées les guinéo-congolaises sont les plus représentées mettent en évidence la prédominance des espèces (37\%) (Figure 4).

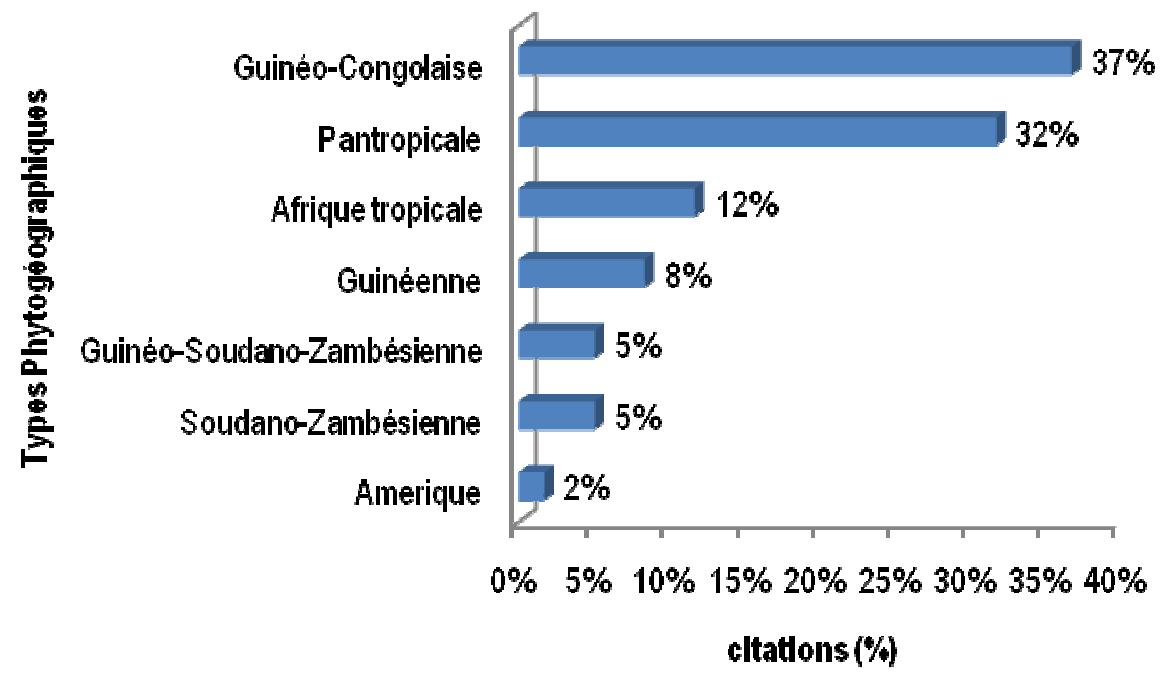

Figure 4 : Spectre pondéré (\% en citation) des types de distributions phytogéographiques.

Les espèces forestières sont les plus représentées parmi les plantes anti hémorroïdaires recensées $(62 \%)$. Elles sont suivies des espèces de culture $(18 \%)$, puis des rudérales (12\%) (Figure 5). Les espèces sarcochores sont les plus représentées $(62 \%)$ et la zoochorie est le mode de dissémination qui prédomine (63\%) (Figure 6). 


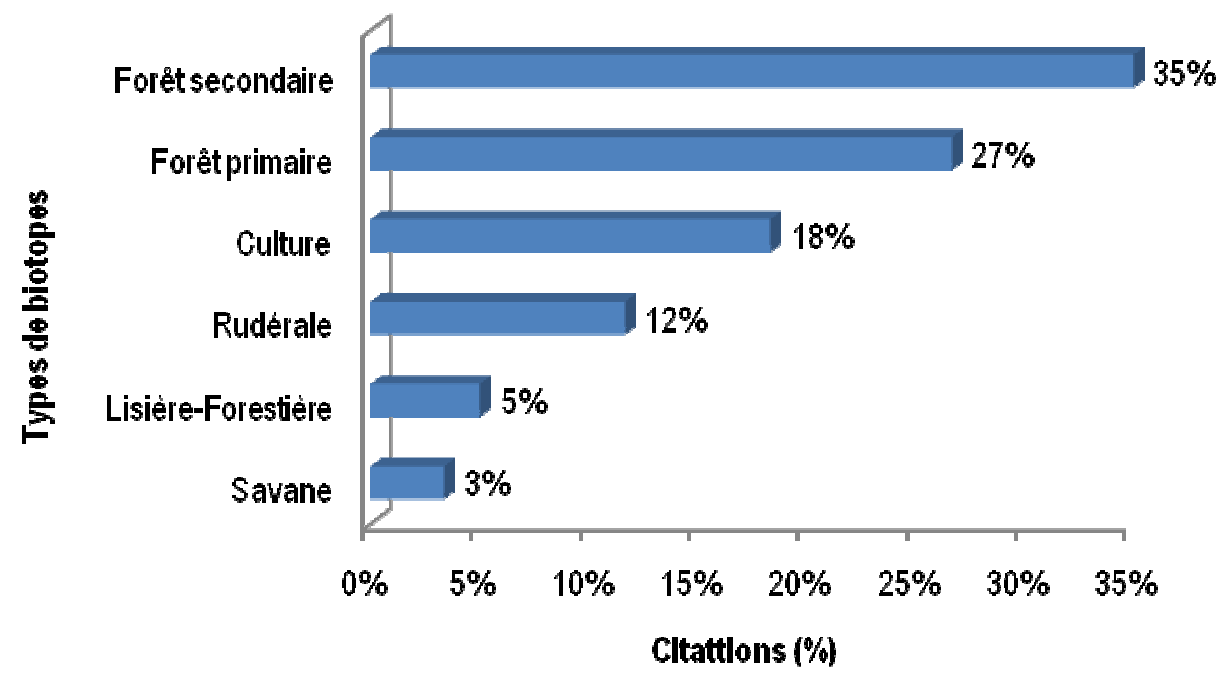

Figure 5 : Spectre pondéré (\% en citation) des types de biotopes.

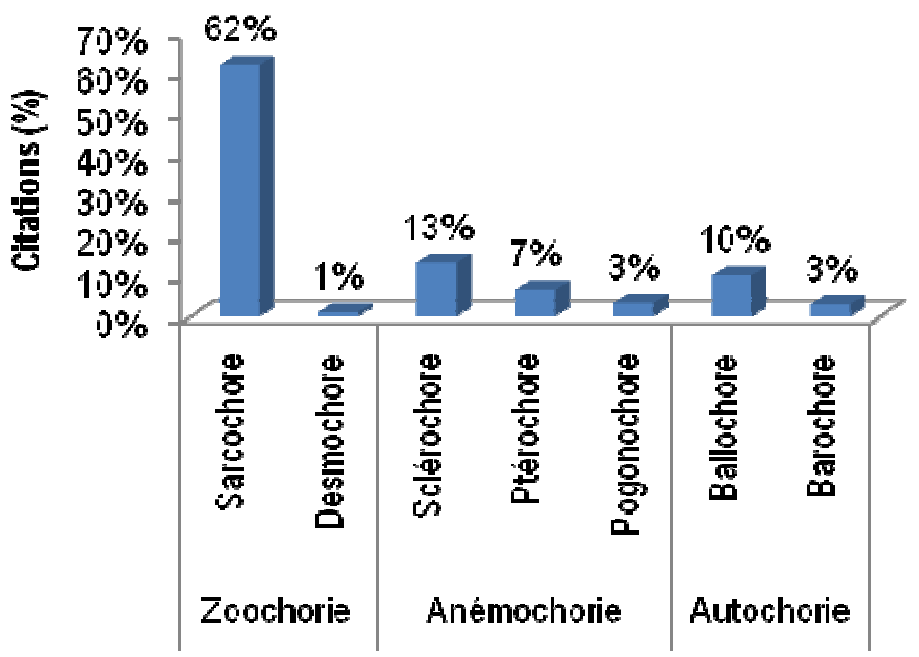

Types ot modes de dissémination des diaspores

Figure 6 : Spectre pondéré (\% en citations) des types et modes de dissémination des diaspores.

Caractérisation des recettes: Des organes prélevés sur le port végétatif des plantes pour la préparation des recettes médicamenteuses, les écorces sont majoritairement utilisées (53\%). Elles sont suivies des feuilles $(20 \%)$ puis des plantes entières (12\%) (Figure 7). 


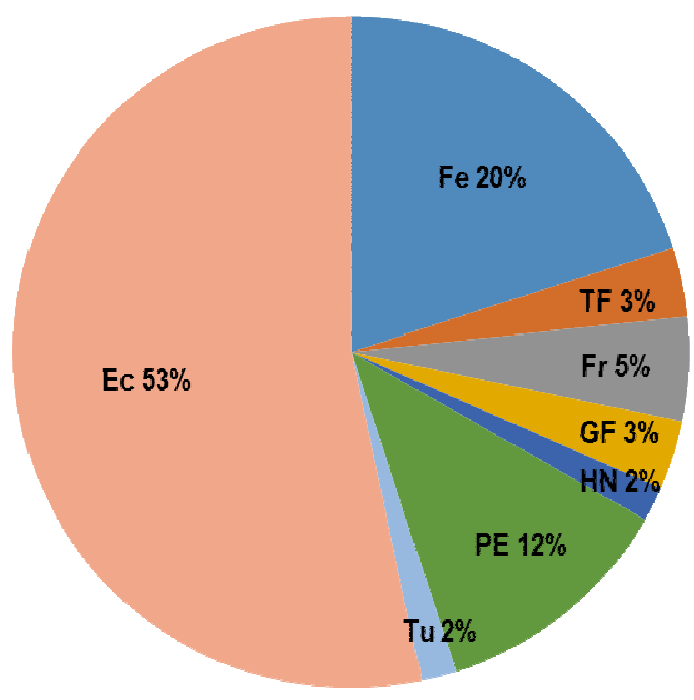

Figure 7: Diagrammes des organes végétatifs des plantes anti hémorroïdaires recensées.

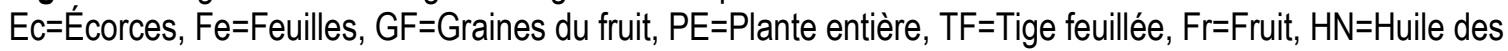
noix, Tu=Tubercule ou rhizome.

La décoction est le mode de préparation le plus employé (57\%). II est suivi du broyage des organes après calcination ou chauffage $(27 \%)$. La macération ne représente que $2 \%$. L'eau $(77 \%)$ est le solvant par excellence pour préparer les recettes contre les hémorroïdes. La boisson prise deux (02) fois par jour plus le bain de siège pris une (01) fois par jour est le mode d'administration des recettes le plus employé par les guérisseurs (47\%). II est suivi de l'emploi des feuilles mélangées à l'huile des noix de palme en suppositoire (22\%). La purge quant à elle ne représente que $7 \%$. Le coût et la durée du traitement varient en fonction du stade et du degré de la maladie. Ainsi, les coûts vont de 5000 à $10000 \mathrm{~F}$ CFA en moyenne selon que la maladie est au Stade I et de 10 à 25000 F CFA lorsque la maladie devient chronique (Stade III). II en est de même pour les durées du traitement ; 3 semaines à 2 mois pour les cas simples, 2 à 5 mois en moyenne pour les cas chroniques.

Facteurs de consensus d'utilisation (ICFs) et Fréquence relative (FRs) des espèces : Le calcul des valeurs de consensus des espèces a relevé des faibles indices de consensus. Aucun indice n'a atteint la valeur moyenne de consensus $(0,5)$. II en est de même pour les fréquences relatives des espèces qui n'atteignent pas les $50 \%$. Huit (08) espèces ont révélé un ICF $\geq 0,10$ et 11 ont atteint une $F R \geq 10 \%$ (Tableau 2).

Tableau 2: Espèces ayant atteint des ICFs et des FRs intéressants.

\begin{tabular}{l|l|l}
\hline Espèces & ICFs & FRs \\
\hline Piper umbellatum & 0,23 & $20,85 \%$ \\
Baillonnella toxisperma & 0,19 & $22,44 \%$ \\
Antrocaryon klaineanum & 0,19 & $20,82 \%$ \\
Irvingia gabonensis & 0,14 & $12,70 \%$ \\
Cylicodiscus gabonensis & 0,14 & $15,03 \%$ \\
Mangifera indica & 0,14 & $14,28 \%$ \\
Pycnanthus angolensis & 0,14 & $15,02 \%$ \\
Morinda lucida & 0,11 & $12,38 \%$ \\
Capsicum frutescens & & $11,38 \%$ \\
Myrianthus arboreus & & $11,12 \%$ \\
Eleais guineensis & & $10,31 \%$ \\
\hline
\end{tabular}


Les espèces ayant obtenu une valeur de consensus supérieure ou égale à 0,1 ont également une fréquence relative supérieure ou égale à $10 \%$ excepté Myrianthus arboreus $(I C F=0,07)$; Capsicum frutescens $(I C F=0,09)$ et Eleais guineensis $(I C F=0,09)$.

Plantes anti hémorroïdaires, maladies soignées et métabolites secondaires : Les plantes anti hémorroïdaires recensées rentrent dans le traitement de nombreuses autres maladies parmi lesquelles les inflammations, les douleurs et les maladies du système digestif qui sont les plus représentées (Tableau 1). La recherche bibliographique des métabolites secondaires révèle que 56 des 60 espèces anti hémorroïdaires recensées contiennent des composés phénoliques tels les tanins $(16 \%)$, les flavonoïdes $(16 \%)$, les polyphénols (11\%). Les saponines sont aussi majoritairement présentes dans les plantes recensées (15\%) (Figure 8).

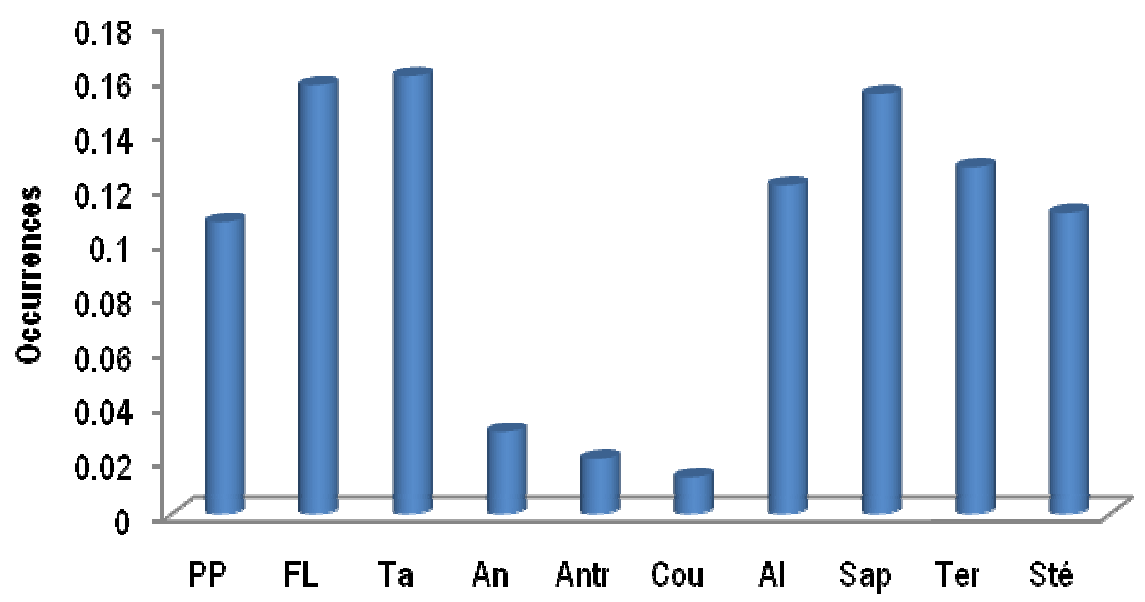

Métabolites secondaires

Figure 8: Histogramme des métabolites secondaires présents dans les plantes recensées.

$\mathrm{PP}=$ Polyphénols, FL=Flavonoïdes, Ta=Tanins, An=Anthocyanes, Antr=Anthraquinones, Cou=coumarines, Al=Alcaloïdes, Sap= Saponines, Ter=Terpènes, Sté=Stérols.

\section{DISCUSSION}

Vingt (20) des plantes anti hémorroïdaires recensées, ont également été identifiées par des auteurs en Afrique. Alchornea cordifolia, Mangifera indica, Capsicum frutescens, Annona senegalensis, Ageratum conyzoïdes, Ocimum gratissimum, Musa paradisiaca et Musanga cecropioides sont utilisées par les pygmées Twa et leur voisin Oto de Bikoro en RDC pour soigner les affections hémorroïdaires (Illumbe et al., 2014). Musa paradisiaca, Ageratum conyzoïdes et Ocimum gratissimum sont également utilisées au Cameroun comme plantes anti hémorroïdaires (Mpondo et al., 2012 ; Ngene et al., 2015). Canarium schweinfurthii et Ficus exasperata sont utilisées par les Kroubou et les populations de Bingerville en Côte d'Ivoire pour soigner les pathologies hémorroïdaires (N'Guessan et al., 2009 ; Séguéna et al., 2013). Entandrophragma cylindricum et Petersianthus macrocarpus ont été citées au Cameroun pour le même usage (Dongmo et al., 2007). II en est de même pour Eremomastax speciosa dans les localités de Fossong-Wentcheng et Foto du Cameroun (Telefo et al., 2011). Psidium guajava, Ricinodendron heudelotii, Adansonia digitata, Irvingia gabonensis et Euphorbia hirta sont également identifiées par Dibong et al. (2011), Yinyang et al. (2014) et Ngene et al. (2015) comme des plantes anti hémorroïdaires. Betti, (2002) trouve également que Pycnanthus angolensis est employée pour le même usage dans les marchés de Yaoundé. Les tradithérapeutes proposent des thérapies diversifiées. L'absence des similitudes traduit le caractère propre à l'ethnobotanique où chaque individus détient un secret qui lui a été transmis soit par ses ancêtres, soit lors de la formation auprès des détenteurs ou alors qu'il a acquis au fil des années d'expérience (Zerbo et al., 2010). 
Cependant, nonobstant le caractère secret de l'ethnobotanique, il peut y avoir des échanges de connaissances entre les tradithérapeutes soit d'une même localité, soit de localités voisines (llumbe et al., 2014). L'exploitation des espèces ligneuses pour soigner les affections hémorroïdaires est préjudiciable car ce sont des espèces très vulnérables (llumbe et al., 2014 ; Kidik Pouka et al., 2015). La distribution phytogéographique de ces espèces témoigne bien du caractère endémique des plantes utilisées ainsi que de la conservation du savoir-faire de la pharmacopée locale (Kidik Pouka et al., 2015). Les tradithérapeutes utilisent les plantes disponibles dans leur biotope leur permettant de disposer des organes nécessaires pour les préparations médicamenteuses à chaque saison de l'année (Zerbo et al., 2010 ; Kidik Pouka et al., 2015). L'importance des espèces zoochores témoigne d'une part de l'origine forestière des plantes anti hémorroïdaires recensées et d'autre part du rôle important de la faune sauvage dans la régénération forestière (Betti, 2002 ; Kidik Pouka et al., 2015). Bien que la différence soit relevée entre hémorroïdes internes et hémorroïdes externes, les guérisseurs ont recourt aux écorces dans les deux cas. La sève de ces écorces aurait un rôle important à jouer dans le traitement des hémorroïdes. C'est elle qui selon les guérisseurs permet de resserrer les tissus des organes. Les feuilles quant à elles sont beaucoup plus sollicitées en cas de prolapsus pour faire rentrer le rectum. L'intérêt porté aux écorces et aux feuilles trouve une explication dans le fait qu'elles sont le siège par excellence de la biosynthèse et même du stockage des métabolites secondaires responsables des propriétés biologiques des plantes (Ngene et al., 2015). Toutefois, si l'utilisation des feuilles n'est guère préjudiciable pour la plante, il n'en demeure pas moins de l'utilisation des écorces. Les informateurs du village Messassi témoignent que l'écorçage excessif d'Erythrophleum suaveolens (Elon) pour soigner les affections hémorroïdaires a fini par tuer le seul arbre qu'il y avait dans le village. II en est de même pour l'écorçage excessif d' Antrocaryon klaineanum (Angongui) au village Nkolmefou. llumbe et al. (2014) trouve que la décoction est le mode de préparation le plus utilisé pour préparer les recettes médicamenteuses, l'eau étant le solvant traditionnel par excellence (Zerbo et al., 2010). Toutefois, l'huile des noix de palme (Eleais guineensis) reste très sollicitée lorsqu'il s'agit des plantes utilisées en suppositoire contre les hémorroïdes externes. La boisson associée au bain de siège est selon llumbe et al. (2014), le mode d'administration par excellence des recettes anti hémorroïdaires. Les guérisseurs soulignent qu'il n'est pas conseillé aux patients atteints d'affections hémorroïdaires de se purger, encore moins lorsque ces hémorroïdes sont externes. Les valeurs des ICFs trouvées sont contraires à celles obtenues par llumbe et al. (2014) qui trouvent de nombreux ICFs présentant une valeur au-dessus de la moyenne $(I C F>0,5)$. Les valeurs élevées des ICFs et des FRs des plantes montrent que ces plantes sont potentiellement actives et donc pourraient présenter un grand intérêt dans le traitement des affections hémorroïdaires. Le faible coût ainsi que la courte durée des traitements de la médecine traditionnelle par rapport à la médecine occidentale seraient pour les populations des pays en voie de développement et ceux des pays industrialisés une aubaine pour venir à bout des affections hémorroïdaires et ainsi accorder une place importante à la médecine traditionnelle. Les guérisseurs des villages identifient les différents stades des pathologies hémorroïdaires ainsi que les troubles qui s'y rattachent (douleurs, rectorragies, marisques, prolapsus, prurits et bourdonnement de ventre) (Faucheron, 2005 ; Boureima, 2006 ; Senéjoux, 2010). Ils utilisent des plantes en association dans la mesure où chacune d'elle a un rôle spécifique à jouer dans le traitement des symptomatologies hémorroïdaires (llumbe et al., 2014). Ainsi, il est précisé que les plantes telles que Baillonella toxisperma, Invingia gabonensis, Mangifera indica, Laportea ovalifolia, Piper umbellatum et Lophira alata jouent le rôle d'anti inflammatoire permettant d'apaiser les douleurs hémorroïdaires. Morinda lucida et Antrocaryon klaineanum faciliteraient la digestion en régulant le transit intestinal, afin d'empêcher au patient atteint d'affections hémorroïdaires d'être constipé pour ainsi lui éviter d'effectuer les efforts de poussée. En cas de rectorragie, Alchornea cordifolia, Canarium schweinfurthii remédieraient aux problèmes d'anémie, Pachyelasma tessmannii, Annona senegalensis et Anchomanes difformis auraient pour rôle de stopper le saignement. Par ailleurs, Pycnanthus angolensis et Spathodea campanulata auraient pour rôle de purifier les vaisseaux étant donné que la congestion et la pression sur une longue durée peuvent entrainer un prolapsus hémorroïdaire (Djahra, 2014 ; Ghedadba et al., 2014). En outre, 
Citrus lemon et Senna alata serviraient à redonner du tonus aux patients affaiblis par la maladie alors que Erythrophleum suaveolens et Ricinodendron heudelotii permettraient de resserrer ou de solidifier les tissus des organes. Les prurits sont généralement dus à des accumulations de déchets et des microbes dans l'organisme d'où le recours aux nettoyeurs tels Cylicodiscus gabonensis, Entandrophragma sp. et Mammea africana. Plusieurs travaux ont montré que toutes ces espèces étaient riches en métabolites secondaires (tanins, flavonoïdes, polyphénols et saponosides) qui sont des composés chimiques responsables de leur efficacité thérapeutique (Dongmo et al., 2007 ; Sereme et al., 2008 ; N'Guessan et al., 2009 ; Kuete et Efferth, 2010 ; Zerbo et al., 2010 ; Kraban et al., 2014). En effet, les tanins ont une activité astringente qui favorise la vasoconstriction, paramètre important dans l'hémostase. Ces effets hémostatique et vasoconstricteur sur les petits vaisseaux, justifient l'utilisation des tanins contre les varices, les hémorroïdes et les blessures superficielles (Biaye, 2002 ; Sereme et al., 2008 ; Ghedadba et al., 2014). Les tanins utilisés par voie orale, assurent une fonction vasoprotectrice. Ils

\section{CONCLUSION}

Les enquêtes ethnobotaniques effectuées auprès des guérisseurs des marchés et villages du Centre et du Littoral du Cameroun révèlent que la flore camerounaise est riche en espèces de plantes médicinales capables de soigner les affections hémorroïdaires. Ces plantes, endémiques pour la plupart, sont utilisées en association et renferment des composés phénoliques en grande quantités, elles seraient pour la médecine moderne d'un intérêt non négligeable afin de venir à bout des

\section{REFERENCES}

Adjanohoun E, 2000. La biodiversité face au développement des industries pharmaceutiques africaines. In Réseau des " espèces ligneuses médicinales ", Eyog Matig O, Adjanohoun E, de Souza S et Sinsin B (eds). Compte rendu de la première réunion du réseau tenue 15-17 décembre 1999 à la station IITA Cotonou, Bénin, 88-103.

Ankouane AF, Kowo M, Ngo Nonga B, Djapa R, Tagni-Sartre M, Njoya O, Ngu Blackett K, Biwolé SM, Ndjitoyap Ndam EC, 2013. Indications, résultats et rendement de la limitent la perte des liquides et favorisent la régénération des tissus (Ghedadba et al., 2014 ; Kraban et al., 2014). Par ailleurs, les flavonoïdes sont reconnus pour leurs nombreuses propriétés biologiques notamment anti-inflammatoire, anticancéreuse, anti radicalaire, anti oxydante puissante et veinotonique (Mpondo et al., 2012 ; Dupuis, 2013). Tous peuvent moduler la transcription d'une enzyme, la cyclo-oxygénase 2 ou COX-2 impliquée dans les processus inflammatoires et cancéreux. Les flavonoïdes de type flavonol et flavonone (rutine, quercétine et ruscogénine) sont utilisés par voie interne pour le traitement de l'insuffisance veineuse et des symptomatologies hémorroïdaires (N'Guessan et al., 2009 ; Djahra, 2014). En outre, les tanins galliques et flavonoïdes sont de puissants laxatifs conseillés en cas de constipation (Ghedadba et al., 2014). Ils calment les coliques et les maux de ventre divers (Sereme et al., 2008). Ce sont avec les polyphénols d'excellents anti diarrhéique et vermifuges (Biaye, 2002 ; Sereme et al., 2008 ; N'Guessan et al., 2009). Les saponosides quant à eux sont de puissants antifongiques qui assurent le nettoyage de l'organisme (Zerbo et al., 2010).

pathologies hémorroïdaires. En outre, le coût réduit des traitements serait pour les populations démunies une alternative dans la lutte contre la pauvreté et contribuerait ainsi à atteindre l'un des objectifs du millénaire (santé pour tous). Toutefois, dans la perspective d'une exploitation durable des espèces, il est impératif de maîtriser les techniques de récoltes des organes des espèces vulnérables très sollicitées.

coloscopie dans un environnement économique défavorable: Cas du Cameroun. Health Sci. Dis. 14: 1-6.

Aubréville A, 1962. Position chorologique du Gabon. Flore du Gabon 2: 3-11.

Awono A, Manirakiza D, Ingram V, 2009. Mobilisation et renforcement des capacités des petites et moyennes entreprises impliquées dans la filière des produits forestiers non ligneux en Afrique Centrale. CIFOR: Cameroun. http: camerounforet.com/fr/bibliotheque/10666. 
Betti JL, 2002. Medicinal plants sold in Yaoundé markets, Cameroon. African Study Monographs 23: 47-64.

Betti JL, Iponga DM, Yongo OD, Mbomio DO, Yobo CM, Ngoye A, 2013. Ethnobotanical study of medicinal plants of the IpassaMakokou Biosphere Reserve, Gabon: Plants used for treating malaria. Journal of Medicinal Plants Research 7: 23002318.

Bézanger-Beauquesne $L$, Pinkas $M$, Torck $M$, 1975. Les plantes dans la thérapeutique moderne. Maloine s.a. éditions, Paris. 529 pp.

Biaye $M$, 2002. Action pharmacologique des tanins. Thèse de Doctorat en Pharmacie, Université Cheikh Anta Diop de Dakar, Sénégal, 53p.

Biyiti LF, Meko'o DJL, Tamzc V, Amvam Zollo PH, 2004. Recherche de l'activité antibactérienne de quatre plantes médicinales camerounaises. Pharm. Med. Trad. Afr. 13: 11-20.

Boureima D, 2006. Connaissances, attitudes et pratiques comportementales liées aux hémorroïdes dans le service de chirurgie générale du CHU Gabriel Touré et auprès des thérapeutes traditionnels au Mali. Thèse de Doctorat, Université de Bamako, Mali, 100p.

Champigneulle B, Dieterling P, Bigard MA, Gaucher P, 1989. Étude prospective de la fonction sphinctérienne anale avant et après hémorroïdectomie. Gastroenterol Clin Biol. 13: 452-456.

Debroux L et Dethier M, 1993. Valorisation des Produits Secondaires de la Forêt dense Tropicale: Réserve de Faune du Dja (Sud-Cameroun). Mémoire présenté en vue de l'obtention du diplôme d'Ingénieur des Eaux et Forêts. Faculté des Sciences Agronomiques, Gembloux, Belgium.

Dibong SD, Mpondo Mpondo E, Ngoye A, Kwin NF, 2011. Plantes médicinales utilisées par les populations bassa de la région de Douala au Cameroun. International Journal of Biological and Chemical Sciences 5: 1105-1117.

Din N, Senger P, Priso JR, Dibong SD, Amougou A, 2002. Logging activities inmangrove forests. A case study of Douala Cameroon. Africa Journal in
Environmental Science and Technology 2: 22-30.

Djahra $A B, 2014$. Étude phytochimique et activité antimicrobienne, antioxydante, antihépatotoxique du Marrube blanc ou Marrubium vulgare L. Thèse de Doctorat Université Badji mokhtar-Annaba, Algérie, 79p.

Dongmo Fomekong GI, Enyong Oben J, Noumessing Momo CE, Enyegue Mandob D, 2007. Phytochemical constituents and Antioxidant potential of some Cameroonian medicinal plants. Pharmacologyonline 2: 436-452.

Dupuis JM, 2013. Prévention et traitement des hémorroïdes. Naturopathie $3 p$.

Evrard C, 1968. Recherches écologiques sur le peuplement forestier des sols hydromorphes de la cuvette centrale Congolaise. Publ. INEAC. Sér. Sc. N¹10 Bruxelles, 295p.

Faucheron JL, 2005. La maladie hémorroïdaire (237a). Alpesmed http://www-sante.ujfgrenoble.fr/SANTEI. $7 \mathrm{p}$.

Fézan $\mathrm{H}$, Tra BI, Guy M, Irié, Kohué CC, N'gaman Clejesson HBM, 2008. Études de quelques plantes thérapeutiques utilisées dans le traitement de l'hypertension artérielle et du diabète: deux maladies émergentes en Côte d'Ivoire. Sciences \& Nature 5: 39-48.

Ghedadba N, Hambaba L, Aberkane MC, OueldMokhtar SM, Fercha N, Bousselsela N, 2014. Évaluation de l'activité hémostatique in vitro de l'extrait aqueux des feuilles de Marrubium vulgare L. Algerian Journal of Natural Products 2: 64-74.

Gnahoue G, Traoré F, Zirihi GN, Guede-Guina F, 2008. Évaluation des effets pharmacodynamiques d'une substance végétale anti hémorroïdaire sur la pression artérielle et artère aorte de mammifères. 9: 6-16.

Golligher JC, 1984. Surgery of the anus, rectum und colon. $5^{\text {th }}$ edn. Bailiere Tindall, London, $113 \mathrm{pp}$.

Haas PA, Fox JR, TA, Haas GP, 1984. The pathogenesis of hemorrhoids. Dis. Colon Rectum 27: 442-50.

Higuero T. 2014. Traitement de la pathologie hémorroïdaire : les nouvelles recommandations. POST'U 1-11. 
llumbe GB, Van Damme P, Lukoki FL, Joiris V, Visser M, Lejoly J, 2014. Contribution à l'étude des plantes médicinales dans le traitement des hémorroïdes par les pygmées Twa et leur voisin Oto de Bikoro, en RDC. Congo Sciences 2: 4654.

Johanson JF et Sonnenberg A, 1990. The prevalence of hemorrhoids and chronic constipation. An epidemiologic study. Gastroenterology 98: 380-386.

Kabran GR, Mamyrbekova-Bekro JA, Pirat JL, Bekro YA, Sommerer N, Verbaere A, Meudec E, 2014. Identification de composés phénoliques extraits de deux plantes de la pharmacopée ivoirienne $\mathrm{J}$. Soc. Ouest-Afr. Chim. 38: 57-63.

Kidik Pouka MC, Ngene JP, Ngoule CC, Mvogo Ottou PB, Ndjib RC, Dibong SD, Mpondo Mpondo E, 2015. Caractérisation des plantes médicinales à flavonoïdes des marchés de Douala (Cameroun). Int. J. Biol. Chem. Sci. 9: 1494-1516.

Kuete V. et Efferth T, 2010. Cameroonian medicinal plants: pharmacology and derived natural products. Frontiers in pharmacology 1: 1-19.

Lehur PA, Gravié JF, Mereutte G, 2001. Circular stapled anopexy for haemorrhoidal disease: results. Colorectal Disease 6: $374-379$

Letouzey R, 1985. Carte phytogéographique du Cameroun au 1/500000 n04. IRAICIV, Université de Toulouse, France, 240p.

Madoff RD et Fleshman JW, 2004. American Gastroenterological Association technical review on the diagnosis and treatment of hemorrhoids. Gastroenterology 126: 1463-1473.

Miller R, Bartolo DC, Roe A, Cervero F, Mortensen NJ, 1988. Anal sensation and the continence mechanism. Dis. Colon Rectum 31: 433-438.

Morgado PJ, Suarez JA, Gomez LG, Morgado JR, 1988. Histoclinical basis for a new classification of hemorrhoidal disease. Dis. Colon Rectum 31: 474-480.

Mpondo Mpondo E, Dibong SD, Ladoh YCF, Priso RJ, Ngoye A, 2012. Les plantes à phénols utilisées par les populations de la ville de Douala. Journal of Animal and Plant Sciences. 15: 2083-2098.
Mullenders W, 1954. La position phytogéographique des hauts plateaux belges. Végétation 5-6: 112-119.

Ngene JP, Ngoule CC, Kidik Pouka CM, Mvogo Ottou PB, Ndjib RC, Dibong SD, Mpondo Mpondo E, 2015. Importance dans la pharmacopée traditionnelle des plantes à flavonoïdes vendues dans les marchés de Douala est (Cameroun). Journal of Applied Biosciences 88: 8194-8210.

N'Guessan K, Kadja B, Guédé N, Zirihi, Traoré D, Aké-assi L, 2009. Screening phytochimique de quelques plantes médicinales ivoiriennes utilisées en pays Krobou (Agboville, Côte-d'Ivoire). Sciences \& Nature 6: 1-15.

Priso RJ, Nnanga JF, Etame J, Din N, Amougou A, 2011. Les produits forestiers non ligneux d'origine végétale: valeur importance dans quelques marchés de la région du Littoral-Cameroun. J. Appl. Biosci. 40: 2715-2726.

République du Cameroun, Stratégie Nationale de Gestion Durable des Eaux et des Sols dans l'espace Agro-Sylvo-Pastoral Au Cameroun (SNGDES). 2007. 89 pp.

Schnell R, 1960. Techniques d'herborisation et de conservation des plantes dans les pays tropicaux. J. Agric. Trop. Bot. Appl. 7: 148.

Sereme A, Millogo-rasolodimby J, Guinko S, Nacro $M, 2008$. Propriétés thérapeutiques des plantes à tanins du Burkina Faso. Pharmacopée et Médecine traditionnelle Africaines 15: 41-49.

Séguéna F, Soro K, Soro D, N'Guessan K, 2013. Savoir -faire des populations locales des taxons du jardin Botanique de Bingerville, Côte d'lvoire. Journal of Applied Biosciences 68 : 5374-5393.

Sénéjoux $A, 2010$. Hémorroïdes. Elsevier Masson SAS 12p.

Shao WJ et Li GC, 2008. Systematic review and meta-analysis of randomized controlled trials comparing stapled haemorrhoïdopexy with conventional haemorrhoidectomy. The Bristish Journal of Surgery 95 : 147-160.

Telefo PB, Lemfack MC, Bayala B, Lienou LL, Goka CS, Yemele MD, Mouokeu C, Tagne SR, Moundipa FP, 2011. Enquête ethnopharmacologique des plantes utilisées dans le traitement de l'infertilité 
féminine dans les localités de FossongWentcheng et Foto, Cameroun. Phytothérapie 11p.

White $F, 1986$. La végétation de l'Afrique. Mémoire accompagnant la carte de végétation de l'Afrique, Unesco/Aetfat/Unso. 384p.

Yinyang J, Mpondo Mpondo E, Tchatat M, Ndjib RC, Mvogo Ottou PB, Dibong SD, 2014. Les plantes à alcaloïdes utilisées par les populations de la ville Douala (Cameroun). Journal of Applied Biosciences 78 : 6600-6619.

Zerbo $\mathrm{P}$, Millogo-Rasolodimby J, NacoulmaOuedraogo OG, Van Damme P, 2007. Contribution à la connaissance des plantes médicinales utilisées dans les soins infantiles en pays San, au Burkina Faso. Int. J. Biol. Chem. Sci. 1: 262-274. 